Article

\title{
Synthesis and Biotransformation of Bicyclic Unsaturated Lactones with Three or Four Methyl Groups
}

\author{
Katarzyna Wińska ${ }^{1, *}$, Małgorzata Grabarczyk ${ }^{1}$, Wanda Mączka ${ }^{1}$, Adrianna Kondas ${ }^{1}$, \\ Gabriela Maciejewska ${ }^{2}$, Radosław Bonikowski ${ }^{3}$ and Mirosław Anioł ${ }^{1}$ \\ 1 Department of Chemistry, Wrocław University of Environmental and Life Sciences, Norwida 25, \\ 50-375 Wrocław, Poland; magrab@onet.pl (M.G.); wanda_m19@o2.pl (W.M.); adakondas@gmail.com (A.K.); \\ miroslaw.aniol@up.wroc.pl (M.A.) \\ 2 Faculty of Chemistry, Wrocław University of Technology, Wybrzeże Wyspiańskiego 27, 50-370 Wrocław, \\ Poland; gabriela.maciejewska@pwr.edu.pl \\ 3 Institute of General Food Chemistry, Lodz University of Technology, Stefanowskiego 4/10, 90-924 Łódź, \\ Poland; radoslaw.bonikowski@p.lodz.pl \\ * Correspondence: katarzyna.winska@upwr.edu.pl; Tel.: +48-71-3205-213
}

Academic Editor: Derek J. McPhee

Received: 21 November 2016; Accepted: 11 January 2017; Published: 17 January 2017

\begin{abstract}
The aim of this study was to obtain new unsaturated lactones by chemical synthesis and their microbial transformations using fungal strains. Some of these strains were able to transform unsaturated lactones into different hydroxy or epoxy derivatives. Strains of Syncephalastrum racemosum and Absidia cylindrospora gave products with a hydroxy group introduced into a tertiary carbon, while the Penicillium vermiculatum strain hydroxylated primary carbons. The Syncephalastrum racemosum strain hydroxylated both substrates in an allylic position. Using the Absidia cylindrospora and Penicillium vermiculatum strains led to the obtained epoxylactones. The structures of all lactones were established on the basis of spectroscopic data.
\end{abstract}

Keywords: unsaturated lactones; hydroxylation; epoxidation; biotransformation

\section{Introduction}

Hydroxylactones are a very important group of natural compounds. Such compounds are often encountered in Nature, mainly in plants and marine organisms. Natural hydroxylactones are widely known for their biological properties, which include cytotoxicity [1-4], anti-cancer effects [5,6], inhibition of plant growth [7,8], anti-inflammatory action $[9,10]$ and anti-depressant properties [10]. Because lactones occur in natural sources in small amounts, their isolation is hard and expensive. From this it follows that in order to study these biologically active compounds it is necessary to obtain them by means other than isolation from their natural sources. One such method is hydroxylation of synthetically obtained lactones by means of biotransformations. Hydroxylation can be performed on different lactone derivatives, one of which are unsaturated lactones. Usually the presence of a double bond in these molecules leads to the introduction of a hydroxy group into the allylic position [11-18]. A double bond in the molecule can also undergo epoxidation [11,15]. Another possibility is the direct hydroxylation of the double bond $[11,13,14,16,18]$ or opening of an epoxide ring $[11,15,18]$. The hydroxy group can also be introduced into primary [19], secondary $[16,19,20]$ or tertiary carbons of the molecules [12,17,20,21].

The biotransformation of lactones with double bonds in their structure may thus yield different and interesting derivatives like hydroxy- or epoxylactones. Our team has been conducting studies dealing with transformations of different kinds of lactones for a long time. Previously unsaturated 
lactones with one, two or three methyl groups attached to the cyclohexane ring were subjected to biotransformation [22-24]. During these biotransformations, hydroxylation in allylic position or epoxidation of double bond was observed. Continuing our research we decided to synthesize two new unsaturated lactones with two or three methyl groups in the cyclohexane ring and one additional methyl group in the lactone ring. We hoped that it would be possible to obtain some interesting hydroxy derivatives as a result of their biotransformation.

\section{Results and Discussion}

New unsaturated lactone biotransformation substrates $\mathbf{5} \mathbf{a}$ and $\mathbf{5} \mathbf{b}$ were obtained from a four-step synthesis from the corresponding known allylic alcohols. Substrates $\mathbf{1 a}$ and $\mathbf{1} \mathbf{b}$ were subjected to a Claisen rearrangement with orthopropionate modification, giving $\gamma, \delta$-unsaturated esters $2 \mathbf{a}$ and $\mathbf{2} \mathbf{b}$ as two pairs of diastereoisomers ( $46 \%$ A:54\% B) and $\mathbf{2 b}(46 \%$ A:54\% B). These esters were then hydrolyzed into the $\gamma, \delta$-unsaturated acids $\mathbf{3 a}$ and $\mathbf{3} \mathbf{b}$ also as pairs of diastereoisomers $(48 \% \mathbf{A}: 52 \% \mathbf{B})$ and $(41 \% \mathbf{A}: 59 \% \mathbf{B})$. In the next step these acids were transformed into the corresponding iodolactones $4 \mathbf{a}$ and $\mathbf{4 b}$. Both iodolactones were also obtained as mixtures of diastereoisomers $4 \mathbf{a}(37 \% \mathbf{A}: 63 \% \mathbf{B})$; $4 \mathbf{b}(39 \% \mathbf{A}: 61 \%$ B). In the last step, the diastereoisomeric mixtures of iodolactones were subjected to dehydrohalogenation, also providing diastereoisomeric mixtures of the unsaturated lactones $\mathbf{5 a}$ $(33 \% \mathbf{A}: 67 \% \mathbf{B})$ and $5 \mathbf{b}(34 \% \mathbf{A}: 66 \% \mathbf{B})$. (Scheme 1$)$.

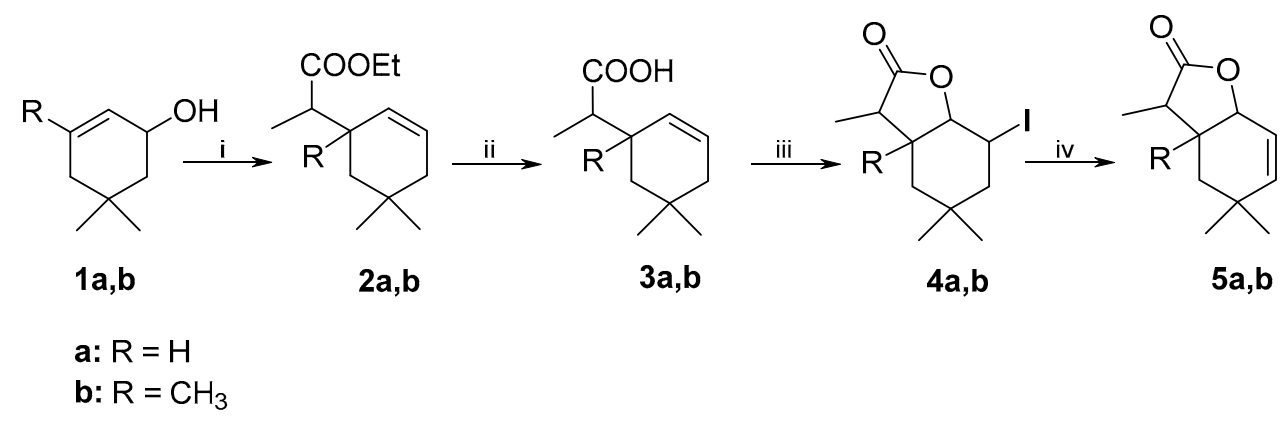

Scheme 1. Synthesis of lactones 5a and 5b. Reagents and Conditions: (i): $\mathrm{CH}_{3} \mathrm{CH}_{2} \mathrm{C}\left(\mathrm{OC}_{2} \mathrm{H}_{5}\right)_{3}$, $\mathrm{CH}_{3} \mathrm{CH}_{2} \mathrm{COOH}, 137{ }^{\circ} \mathrm{C}$ (2a: 84\%, 2b: 82\%); (ii): $\mathrm{KOH}, \mathrm{EtOH}, 100{ }^{\circ} \mathrm{C}$ (3a: 96\%, 3b: 92\%); (iii): $\mathrm{I}_{2}$, KI r.t. (4a: $71 \%$, 4b: $75 \%)$; (iv): DBU, toluene, $110{ }^{\circ} \mathrm{C}(5 \mathbf{a}: 79 \%, 5 \mathbf{b}: 79 \%)$.

The structures of these compounds were established on the basis of their spectral data $\left({ }^{1} \mathrm{H}-\mathrm{NMR}\right.$, $\left.{ }^{13} \mathrm{C}-\mathrm{NMR}, \mathrm{COSY}, \mathrm{HMQC}, \mathrm{IR}\right)$ and confirmed by HRMS analysis. Because the aim of our study was to obtain new lactones, these compounds will be discussed in detail here. In the case of iodolactone $\mathbf{4 b}$ and unsaturated lactone $\mathbf{5 b}$, we were able to partially separate each of the diastereoisomers in small quantities which allowed for a more accurate analysis of these compounds. Analysis of the ${ }^{1} \mathrm{H}-\mathrm{NMR}$ spectra of iodolactones $\mathbf{4 a}$ and $\mathbf{4 b}$ indicates the presence of two diastereoisomers named $\mathbf{A}$ and $\mathbf{B}$ Firstly the spectrum of iodolactone $\mathbf{4 a}$ (as mixture of diastereoisomers $\mathbf{A}$ and $\mathbf{B}$ ) will be discussed. Both signals of the H-1 protons appear as narrow singlets, suggesting an equatorial orientation of these protons. Signals corresponding to the H-2 and H-6 protons are broad multiplets, indicating their axial orientation. It follows from this that the $\mathrm{C}-\mathrm{O}$ bonds in both molecules occupy an axial position. The $\mathrm{CH}_{3}-11$ groups are located in the same plane as the lactone ring (4a-A) or across this plane (4a-B). In the case of iodolactone $4 \mathrm{~b}$ it was possible to analyze each diastereoisomer separately. In the case of compound $\mathbf{4 b - A}$, the signal coming from proton $\mathrm{H}-2$ is a wide multiplet, suggesting its axial orientation. The doublet with a smaller $(5.0 \mathrm{~Hz})$ coupling constant corresponding to the $\mathrm{H}-1$ proton indicates its equatorial orientation. A different situation is observed in the case of compound 4b-B. Both signals of the $\mathrm{H}-1(\mathrm{~d}, J=10.2 \mathrm{~Hz})$ and $\mathrm{H}-2(\mathrm{ddd}, J=13.9,10.2$ and $3.8 \mathrm{~Hz}$ ) protons suggested their axial orientation. These observations indicated that in compound $4 \mathbf{b}-\mathbf{A}$, the $\mathrm{C}-\mathrm{O}$ bond of the lactone ring occupied an axial position, but in compound $\mathbf{4 b - B}$ this bond is in an equatorial position. 
In the case of isomer $4 \mathbf{b}-\mathbf{A}$, the $\mathrm{CH}_{3}-11$ group is located across the plane of the lactone ring and in the case of isomer $\mathbf{4 b - B}$ this group is lying in the same plane as the lactone ring (Figure 1).
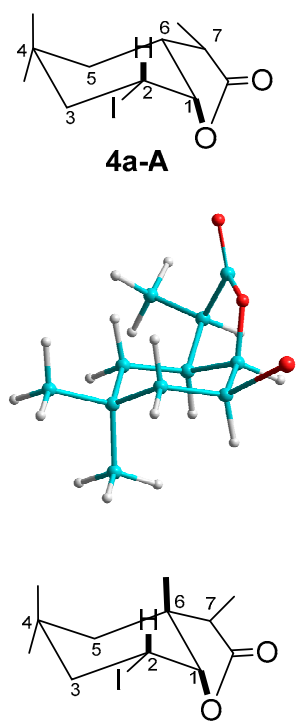

4b-A

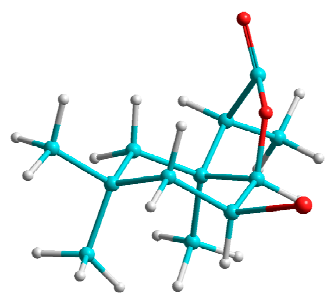

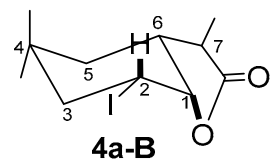

4a-B
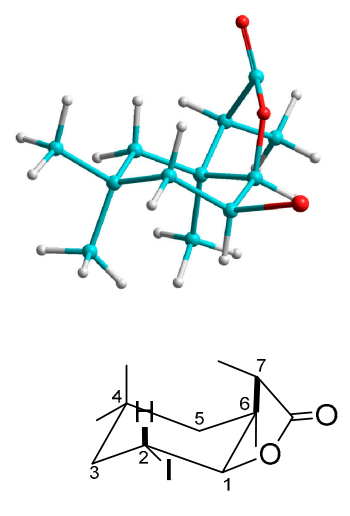

$4 b-B$

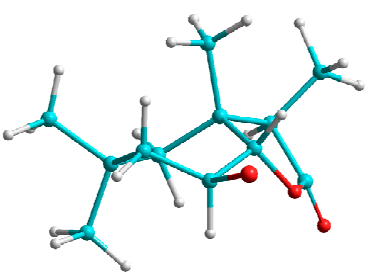

Figure 1. Structures of iodolactones $4 \mathbf{a}$ and $\mathbf{4 b}$.

Analysis of the ${ }^{1} \mathrm{H}$-NMR spectra of the unsaturated lactones $\mathbf{5 a}$ and $\mathbf{5} \mathbf{b}$ proves that these compounds are also mixtures of two diastereoisomers named $\mathbf{A}$ and $\mathbf{B}$. The structures of these lactones are very similar to those described above for the corresponding iodolactones.

The only difference is the presence of a double bond between carbons C-2 and C-3. The signals of the $\mathrm{H}-2$ and $\mathrm{H}-3$ protons, which look like wide multiplets for lactone $\mathbf{5 a}$, suggests their axial orientation. In the case of lactone $\mathbf{5 b}$, the coupling constant between protons $\mathrm{H}-2$ and $\mathrm{H}-3$ is $10.2 \mathrm{~Hz}$ (for $\mathbf{5 b}-\mathbf{A}$ ) and $10.0 \mathrm{~Hz}$ (for $\mathbf{5 b}-\mathbf{B}$ ), also indicating their axial position (Figure 2).

Both unsaturated lactones $\mathbf{5 a}$ and $\mathbf{5 b}$ (as mixtures of diastereoisomers) were used as biotransformation substrates. During the screening biotransformation, the ability of some fungal strains to convert lactones $\mathbf{5 a}$ and $\mathbf{5 b}$ into any products was checked. From the fourteen different strains (Fusarium sp., Penicilium sp., Absidia sp. and Syncephalastrum racemosum) examined only some of them showed any ability to transform the substrates into some derivatives. These positive results obtained during screening biotransformation using these five strains are presented in Tables 1 and 2.

As it can be seen in the above tables, the unsaturated lactones $\mathbf{5 a}$ and $\mathbf{5 b}$ were converted into different products by three (5a) or five strains $(\mathbf{5 b})$, respectively Taking into consideration the yields of these processes, the strains S. racemosum AM105, A. cylindrospora AM336 and P. vermiculatum AM30 were chosen for preparative-scale biotransformations of both substrates. The results of this are presented in Tables 3 and 4. 

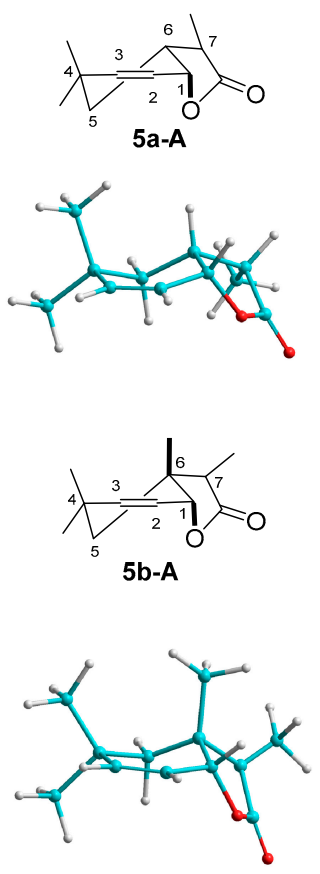
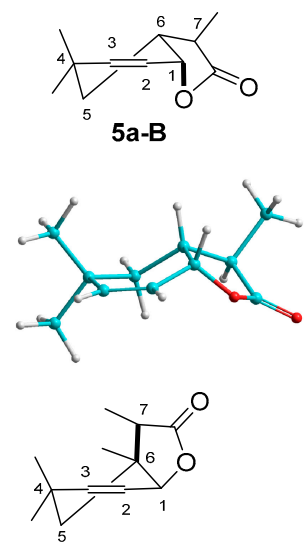

$5 b-B$

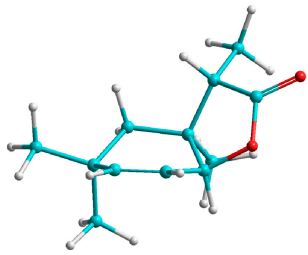

Figure 2. Structure of unsaturated lactones $5 \mathbf{a}$ and $\mathbf{5 b}$.

Table 1. Positive results of the screening biotransformation of lactone $5 \mathbf{a}$ after 7 and 14 days of incubation (in \% according to GC).

\begin{tabular}{ccccccc}
\hline Strain & Days & $\begin{array}{c}\text { Lactone 5a } \\
\mathbf{( A + B )}\end{array}$ & $\begin{array}{c}\text { Lactone 6a } \\
\mathbf{( A + \mathbf { B } )}\end{array}$ & $\begin{array}{c}\text { Lactone 7a } \\
\mathbf{( B )}\end{array}$ & $\begin{array}{c}\text { Lactone 8a } \\
\mathbf{( B )}\end{array}$ & $\begin{array}{c}\text { Lactone 9a } \\
\mathbf{( A +} \mathbf{B})\end{array}$ \\
\hline $\begin{array}{c}\text { S. racemosum } \\
\text { AM105 }\end{array}$ & 7 & $9.7+70.2$ & $0+9.3$ & 10.8 & - & - \\
\hline A. cylindrospora & 7 & $0+36.5$ & $10.2+25.0$ & 24.5 & - & - \\
AM336 & 14 & $11.1+70.3$ & - & 15.0 & - & - \\
\hline A. glauca & 7 & $12.2+70.8$ & $0+12.3$ & 4.6 & - & - \\
AM177 & 14 & $9.2+54.9$ & $5.6+20.4$ & 9.9 & - & - \\
\hline A. glauca & 7 & $11.3+57.9$ & $4.8+13.9$ & 12.0 & - & - \\
AM254 & 14 & $9.6+57.8$ & $3.5+13.3$ & 15.8 & - & - \\
\hline P. vermiculatum & 7 & - & - & - & 82.6 & - \\
AM30 & 14 & - & - & - & 87.1 & - \\
\hline
\end{tabular}

Table 2. Positive results of the screening biotransformation of lactone $5 \mathbf{b}$ after 7 and 14 days of incubation (in \% according to GC).

\begin{tabular}{|c|c|c|c|c|c|c|}
\hline Strain & Days & $\begin{array}{c}\text { Lactone } 5 b \\
(A+B)\end{array}$ & $\begin{array}{l}\text { Lactone } 6 b \\
(A+B)\end{array}$ & $\begin{array}{l}\text { Lactone } 7 b \\
\text { (B) }\end{array}$ & $\begin{array}{l}\text { Lactone } 8 b \\
\text { (B) }\end{array}$ & $\begin{array}{c}\text { Lactone } 9 b \\
(A+B)\end{array}$ \\
\hline S. racemosum & 7 & $22.3+48.0$ & $0+7.9$ & 21.8 & - & - \\
\hline AM105 & 14 & $11.1+22.6$ & $8.6+19.7$ & 38.0 & - & - \\
\hline A. cylindrospora & 7 & $24.4+54.6$ & - & 21.0 & - & - \\
\hline AM336 & 14 & $25.9+33.2$ & - & 50.9 & - & - \\
\hline A. glauca & 7 & $21.7+44.6$ & $8.4+16.3$ & 9.0 & - & - \\
\hline AM177 & 14 & $18.0+35.5$ & $8.1+22.6$ & 15.8 & - & - \\
\hline A. glauca & 7 & $36.8+45.8$ & $4.9+4.6$ & 7.9 & - & - \\
\hline AM254 & 14 & $24.7+31.4$ & $9.9+9.4$ & 14.5 & - & - \\
\hline P. vermiculatum & 7 & $10.9+40.8$ & - & - & 16.2 & $25.9+6.2$ \\
\hline AM30 & 14 & $0+8.3$ & - & - & 36.7 & $43.8+10.9$ \\
\hline
\end{tabular}


Table 3. Results of the preparative-scale biotransformation of lactone 5 a after 14 days of incubation (in \% according to GC).

\begin{tabular}{cccccc}
\hline Strain & $\begin{array}{c}\text { Lactone } \\
\mathbf{5 a} \mathbf{( A + B )}\end{array}$ & $\begin{array}{c}\text { Lactone } \\
\mathbf{6 a} \mathbf{( A + \mathbf { B } )}\end{array}$ & $\begin{array}{c}\text { Lactone } \\
\mathbf{7 a} \mathbf{( B )}\end{array}$ & $\begin{array}{c}\text { Lactone 8a } \\
\mathbf{( B )}\end{array}$ & $\begin{array}{c}\text { Lactone 9a } \\
\mathbf{( A + \mathbf { B } )}\end{array}$ \\
\hline S. racemosum AM105 & $0+31.2$ & $13.0+37.5$ & 18.4 & - & - \\
A. cylindrospora AM336 & $14.7+30.1$ & - & 27.5 & - & $16.2+11.4$ \\
P. vermiculatum AM30 & $0+16.2$ & - & 40.4 & 43.4 & - \\
\hline
\end{tabular}

Table 4. Results of the preparative-scale biotransformation of lactone $5 \mathbf{b}$ after 14 days of incubation (in \% according to GC).

\begin{tabular}{cccccc}
\hline Strain & $\begin{array}{c}\text { Lactone } 5 \mathbf{b} \\
\mathbf{( A + \mathbf { B } )}\end{array}$ & $\begin{array}{c}\text { Lactone 6b } \\
\mathbf{( A + \mathbf { B } )}\end{array}$ & $\begin{array}{c}\text { Lactone } 7 \mathbf{b} \\
\mathbf{( B )}\end{array}$ & $\begin{array}{c}\text { Lactone } 8 \mathbf{b} \\
\mathbf{( B )}\end{array}$ & $\begin{array}{c}\text { Lactone } \mathbf{9 b} \\
\mathbf{( A +}+\mathbf{B})\end{array}$ \\
\hline S. racemosum AM105 & $8.4+14.5$ & $9.1+33.4$ & 34.6 & - & - \\
A. cylindrospora AM336 & $20.4+36.5$ & - & 43.1 & - & - \\
P. vermiculatum AM30 & $0+6.1$ & - & - & 48.7 & $23.6+21.6$ \\
\hline
\end{tabular}

The biotransformation results proved that each substrate was converted into four different products. One of them-lactone $7 \mathbf{a}$-was produced by all three fungal strains. Its analog-lactone $\mathbf{7 b}$-by two strains (not by P. vermiculatum). Lactones $\mathbf{8 a}$ and $\mathbf{8} \mathbf{b}$ were formed by the P. vermiculatum strain. It is worth noting that these products were formed as single isomers. The S. racemosum strain was able to produce lactones $\mathbf{6 a}$ and $\mathbf{6 b}$ as pairs of diastereoisomers. A similar situation (the formation of products observed as pairs of diastereoisomers) was observed in the case of lactones $9 \mathbf{a}$ and $\mathbf{9 b}$ (Scheme 2).

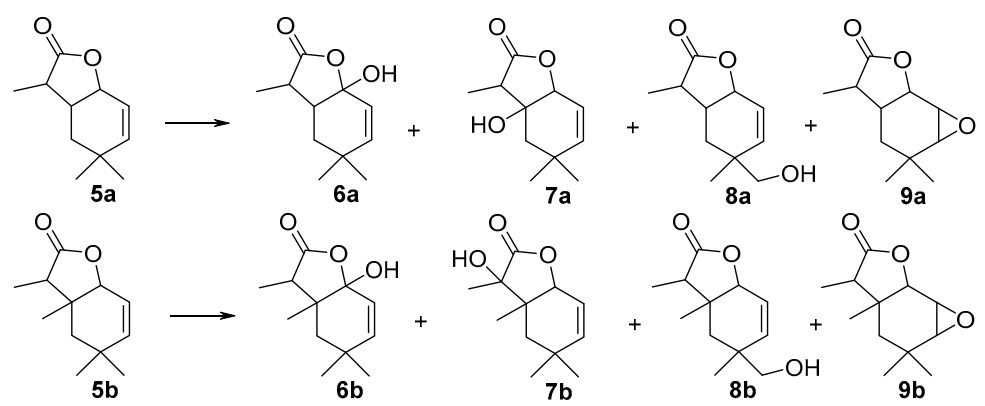

Scheme 2. The products of the biotransformation of unsaturated lactones $\mathbf{5 a}$ and $\mathbf{5 b}$.

After the separation and purification of all compounds obtained during the preparative-scale biotransformations of lactones $\mathbf{5 a}$ and $\mathbf{5 b}$ it was possible to determine the quantity of each of them. These data are listed in Tables 5 and 6.

Table 5. Yield of products obtained from lactone 5a.

\begin{tabular}{ccccc}
\hline Strain & $\begin{array}{c}\text { Lactone 6a (A+ B) } \\
\mathbf{m g / \%}\end{array}$ & $\begin{array}{c}\text { Lactone 7a (B) } \\
\mathbf{m g} / \%\end{array}$ & $\begin{array}{c}\text { Lactone 8a (B) } \\
\mathbf{m g} / \%\end{array}$ & $\begin{array}{c}\text { Lactone 9a (A + B) } \\
\mathbf{m g} / \%\end{array}$ \\
\hline S. racemosum AM105 & $21.7 / 19.9$ & $13.7 / 12.6$ & - & - \\
A. cylindrospora AM336 & - & $15.8 / 14.5$ & - & $8.1 / 7.4$ \\
P. vermiculatum AM30 & - & $29.2 / 26.8$ & $35.7 / 32.8$ & - \\
\hline
\end{tabular}

Analysis of the ${ }^{1} \mathrm{H}-\mathrm{NMR}$ spectra of all obtained products proved that the microorganisms used here preferred hydroxylation of the tertiary carbons present in the molecules of both substrates. All three fungal strains converted substrate $\mathbf{5 a}$ into hydroxylactone $\mathbf{7 a}$, in which the hydroxy group attacks the tertiary C-6 carbon. A comparison of the ${ }^{1} \mathrm{H}-\mathrm{NMR}$ spectra of the substrate with the product 
concluded that this product was created only from isomer $\mathbf{B}$ of the substrate. This result was very surprising because until now such a hydroxylation position was not observed in similar compounds. The $S$. racemosum strain was able to introduce the hydroxy group in an allylic position (the tertiary C-1 carbon) giving product $6 \mathbf{a}$ as a pair of diastereoisomers, whereas in the case of the P. vermiculatum strain, the product 8a in which the hydroxy group is introduced into the $\mathrm{CH}_{3}-10$ group was obtained. This compound was created only from isomer $\mathbf{B}$ of the substrate. The A. cylindrospora strain gave one other product-the epoxylactone $\mathbf{9 a}$ - as a pair of diastereoisomers. The small coupling constants between protons $\mathrm{H}-1, \mathrm{H}-2$ and $\mathrm{H}-3$ (about $3.0 \mathrm{~Hz}$ ) in epoxylactone 9a indicate that these protons are in equatorial positions. This observation also indicates that the epoxide ring is introduced trans relative to the lactone ring.

Table 6. Yield of products obtained from lactone $5 \mathbf{b}$.

\begin{tabular}{ccccc}
\hline Strain & $\begin{array}{c}\text { Lactone } \mathbf{6 b} \mathbf{b}+\mathbf{A}) \\
\mathbf{m g} / \mathbf{\%}\end{array}$ & $\begin{array}{c}\text { Lactone } \mathbf{7 b}(\mathbf{b}) \\
\mathbf{m g} / \%\end{array}$ & $\begin{array}{c}\text { Lactone } 8 \mathbf{b}(\mathbf{B}) \\
\mathbf{m g} / \%\end{array}$ & $\begin{array}{c}\text { Lactone } \mathbf{9 b}(\mathbf{A}+\mathbf{B}) \\
\mathbf{m g} / \%\end{array}$ \\
\hline S. racemosum AM105 & $19.6 / 18.1$ & $11.4 / 10.5$ & - & - \\
A. cylindrospora AM336 & - & $19.8 / 18.3$ & - & - \\
P. vermiculatum AM30 & - & - & $19.0 / 17.6$ & $13.4 / 12.4$ \\
\hline
\end{tabular}

In the case of lactone $\mathbf{5 b}$ some other products were also observed. Two strains (S. racemosum and A. cylindrospora) transformed this compound into lactone $\mathbf{7 b}$, in which the hydroxy group is introduced into the tertiary C-7 carbon. Taking into consideration the spatial structure of both diastereoisomers, it can be observed that for the above product, isomer $\mathbf{B}$ of the substrate is more privileged. In this case hydroxylation of carbon C-6 was not possible, because this position is occupied by the methyl group. Therefore the hydroxy group was introduced into the next tertiary carbon (C-7). Like in substrate $\mathbf{5 a}$, the S. racemosum strain introduced the hydroxy group in an allylic position giving product $\mathbf{6 b}$, also as a pair of diastereoisomers. The P. vermiculatum strain in this case produced two products-hydroxylactone $\mathbf{8 b}$ with the hydroxy group introduced into the $\mathrm{CH}_{3}-10$ group and the epoxylactone $\mathbf{9 b}$. The first of them was created only from isomer $\mathbf{B}$ of the substrate, while the second one was a pair of diastereoisomers. In epoxylactone $\mathbf{9 b}$ a small (about $3.0 \mathrm{~Hz}$ ) coupling constant is observed between protons $\mathrm{H}-1, \mathrm{H}-2$ and $\mathrm{H}-3$. This information suggests the equatorial positions of these protons and also the trans orientation of the epoxide ring in relation to the lactone ring.

During these transformations the formation of two kinds of products, either as a single isomer (B) or as a pair of diastereoisomers $(\mathbf{A}+\mathbf{B})$, was observed. This was because isomer $\mathbf{B}$ was the predominant one in the substrate mixtures.

In the next step, enantiospecificity and optical purity of hydroxylactone $\mathbf{7 a - B}, \mathbf{7 b}-\mathbf{B}, \mathbf{8} \mathbf{a}-\mathbf{B}$ and $\mathbf{8 b}-\mathbf{B}$ which were obtained as single isomers during the preparative biotransformations, were determined. The results of this step are presented in Table 7.

Table 7. The values of enantiospecificity and optical purity of hydroxylactones $7 \mathbf{a}-\mathbf{B}, \mathbf{7 b}-\mathbf{B}, \mathbf{8 a - B}$ and $8 \mathbf{b}-\mathbf{B}$.

\begin{tabular}{cccc}
\hline Strain & Lactone & ee (\%) & {$[\boldsymbol{\alpha}]_{\mathbf{2 0}}^{D}$} \\
\hline \multirow{2}{*}{ S. racemosum AM105 } & 7a-B & 47.9 & $-23.8\left(c=0.51, \mathrm{CH}_{3} \mathrm{Cl}\right)$ \\
& 7b-B & 50.8 & $+10.1\left(c=0.60, \mathrm{CH}_{3} \mathrm{Cl}\right)$ \\
\hline \multirow{2}{*}{ A. cylindrospora AM336 } & 7a-B & 72.1 & $-27.4\left(c=0.70, \mathrm{CH}_{3} \mathrm{Cl}\right)$ \\
& 7b-B & 57.2 & $+15.3\left(c=0.45, \mathrm{CH}_{3} \mathrm{Cl}\right)$ \\
\hline \multirow{3}{*}{ P. vermiculatum AM30 } & 7a-B & 36.8 & $-18.0\left(c=0.58, \mathrm{CH}_{3} \mathrm{Cl}\right)$ \\
& $\mathbf{8 a - B}$ & 34.7 & $+17.2\left(c=0.67, \mathrm{CH}_{3} \mathrm{Cl}\right)$ \\
& $\mathbf{8 b - B}$ & 38.5 & $+21.1\left(c=0.35, \mathrm{CH}_{3} \mathrm{Cl}\right)$ \\
\hline
\end{tabular}


The best enantiomeric excess (72.1\%) was observed for this compound when A. cylindrospora was used as a biocatalyst. In the case of lactone $\mathbf{7 a - B}$ the $(-)$-isomer was preferentially formed. The other compounds, lactones $\mathbf{7 b - B}, \mathbf{8} \mathbf{a}-\mathbf{B}$ and $\mathbf{8 b}-\mathbf{B}$, were created with a predominance of the (+)-isomer.

\section{Materials and Methods}

\subsection{General Methods}

The progress of the reactions and biotransformation was checked by analytical TLC on silica gel-coated aluminium plates (DC-Alufolien Kieselgel 60 F254, Merck, Darmstadt, Germany) with a mixture of hexane and acetone in various ratios as eluent. Preparative column chromatography was performed on silica gel (Kieselgel 60, 230-400 mesh ASTM, Merck) with a mixture of hexane and acetone (for esters hexane-acetone 19:1, for acids hexane-acetone 3:1, for iodolactones and unsaturated lactones hexane-acetone 6:1, for biotransformations products hexane-acetone 3:1) as eluents. Compounds were detected by spraying the plates with $1 \% \mathrm{Ce}\left(\mathrm{SO}_{4}\right)_{2}, 2 \% \mathrm{H}_{3}\left[\mathrm{P}\left(\mathrm{Mo}_{3} \mathrm{O}_{10}\right)_{4}\right]$ in $10 \% \mathrm{H}_{2} \mathrm{SO}_{4}$ or $20 \%$ ethanolic $\mathrm{H}_{2} \mathrm{SO}_{4}$, containing $0.1 \%$ anisaldehyde, followed by heating to $120^{\circ} \mathrm{C}$. GC analysis was carried out on an Agilent Technologies 6890N instrument (Agilent Technologies, Santa Clara, CA, USA) using a DB-17 column (cross-linked methyl silicone gum, $30 \mathrm{~m} \times 0.32 \mathrm{~mm} \times 0.25 \mu \mathrm{m}$ ). The enantiomeric compositions of the products obtained during the biotransformation were determined by Agilent Technologies $6890 \mathrm{~N}$ GC analysis using the chiral column CP-cyclodextrin-B-110 (30 $\mathrm{m} \times 0.25 \mathrm{~mm} \times 0.25 \mu \mathrm{m})$ (Supelco, Bellefonte, PA, USA) under the following conditions: injector $200{ }^{\circ} \mathrm{C}$, detector (FID) $200{ }^{\circ} \mathrm{C}$, column temperature $160{ }^{\circ} \mathrm{C}$, ramp $160-175{ }^{\circ} \mathrm{C}$ at a rate of $0.5^{\circ} \mathrm{C} / \mathrm{min}$, ramp $175-200{ }^{\circ} \mathrm{C}$ at a rate of $10{ }^{\circ} \mathrm{C} / \mathrm{min}$ and hold $1 \mathrm{~min}$ at $200^{\circ} \mathrm{C}$ (for compounds 7a, 8a, 8b) (Supplementary Materials, Figures S110-S107, S113). For compound 7b enantiomeric excess was determined by a Varian CP3380 instrument (Varian, Agilent Technologies, Santa Clara, CA, USA) using a Gamma DEX $325(30 \mathrm{~m} \times 0.25 \mathrm{~mm} \times 0.25 \mu \mathrm{m})$ chiral column (Supelco) (injector $150{ }^{\circ} \mathrm{C}$, detector (FID) $200{ }^{\circ} \mathrm{C}$, column temperature $130{ }^{\circ} \mathrm{C}$, ramp $130-175{ }^{\circ} \mathrm{C}$ at a rate of $0.7^{\circ} \mathrm{C} / \mathrm{min}$, ramp $175-200^{\circ} \mathrm{C}$ at a rate of $20^{\circ} \mathrm{C} / \mathrm{min}$ ) (Supplementary Materials, Figures S111 and S112). The molar masses of the obtained compounds were confirmed by high resolution mass spectrometry analysis using a Waters LCT Premier XE instrument (ESI ionization, Waters, Milford, MA, USA). NMR spectra were recorded in a $\mathrm{CDCl}_{3}$ solution on an Avance ${ }^{\mathrm{TM}} 600 \mathrm{MHz}$ spectrometer $\left(600 \mathrm{MHz}\right.$ for ${ }^{1} \mathrm{H}, 151 \mathrm{MHz}$ for ${ }^{13} \mathrm{C}$, Bruker, Billerica, MA, USA). Chemical shifts are reported in reference to the residual solvent signal $\left(\delta_{\mathrm{H}}=7.26\right)$. IR spectra were recorded on a IR300 FT-IR spectrometer (Thermo-Nicolet, Waltham, MA, USA). Optical rotations were determined on a P-2000 polarimeter (Jasco, Easton, PA, USA) in chloroform solutions, with concentrations denoted in $\mathrm{g} / 100 \mathrm{~mL}$. The melting points were determined on a Boetius apparatus. The refractive index was measured on a Carl Zeiss Abbe and Pulfrich refractometer (Carl Zeiss AG, Jena, Germany).

\subsection{Synthesis of Substrates}

Two known allylic alcohols $\mathbf{1 a}$ [25] and $\mathbf{1 b}$ [26] were used as the starting materials for obtaining unsaturated lactones. All of the intermediates were obtained according to the procedures described below:

3.2.1. Ethyl (5,5,7-Trimethylcyclohex-2-en-1-yl)acetate (2a) and Ethyl (1,5,5,7-tetramethylcyclohex2-en-1-yl)acetate (2b)

Ester 2a (5.0 g, $23.8 \mathrm{mmol}$, yield $84 \%)$ and $\mathbf{2 b}(5.0 \mathrm{~g}, 22.3 \mathrm{mmol}$, yield $82 \%)$ were obtained as mixtures of two diastereoisomers from the Claisen rearrangement with orthopropionate modification [27]. They displayed the following physical and spectral properties:

2a: $\mathrm{n}_{\mathrm{D}}=1.4571 ;{ }^{1} \mathrm{H}-\mathrm{NMR}: 0.91$ (s, 3H, CH$\left.-9 \mathrm{~B}\right), 0.92$ (s, 3H, $\left.\mathrm{CH}_{3}-9 \mathrm{~A}\right), 0.96$ (s, 3H, CH $\left.3-10 \mathrm{~A}\right), 0.97$ (s, 3H, $\left.\mathrm{CH}_{3}-10 \mathrm{~B}\right), 1.12\left(\mathrm{~d}, J=7.0 \mathrm{~Hz}, 3 \mathrm{H}, \mathrm{CH}_{3}-11 \mathrm{~A}\right), 1.16\left(\mathrm{~d}, J=7.0 \mathrm{~Hz}, 3 \mathrm{H}, \mathrm{CH}_{3}-11 \mathrm{~B}\right), 1.27(\mathrm{dd}, J=7.0$ and $\left.3.2 \mathrm{~Hz}, 3 \mathrm{H}, \mathrm{CH}_{3}-12 \mathrm{~B}\right), 1.29\left(\mathrm{dd}, J=7.2\right.$ and $\left.3.2 \mathrm{~Hz}, 3 \mathrm{H}, \mathrm{CH}_{3}-12 \mathrm{~A}\right), 1.33-1.34(\mathrm{~m}, 1 \mathrm{H}, \mathrm{H}-1 \mathrm{~B}), 1.35-1.36(\mathrm{~m}$, 1H, H-1A), 1.70-1.74 (m, 2H, $\left.\mathrm{CH}_{2}-4 \mathrm{~A}\right), 1.84-1.87$ (m, 2H, $\left.\mathrm{CH}_{2}-4 \mathrm{~B}\right), 2.33-2.36$ (m, 2H, $\left.\mathrm{CH}_{2}-6 \mathrm{~B}\right), 2.39-2.42$ 
(m, 2H, CH $\left.\mathrm{CH}_{2}-6 \mathrm{~A}\right), 2.44-2.48$ (m, 1H, $\left.\mathrm{CH}_{2}-6 \mathrm{~A}\right), 2.49-2.52$ (m, 2H, $\left.\mathrm{CH}_{2}-7 \mathrm{~A}, \mathrm{CH}_{2}-7 \mathrm{~B}\right), 4.16-4.19$ (m, 4H, $\left.\mathrm{CH}_{2}-12 \mathrm{~A}, \mathrm{CH}_{2}-12 \mathrm{~B}\right), 5.44-5.46$ (m, 1H, H-2B), 5.58-5.60 (m, 1H, H-2A), 5.66-5.70 (m, 2H, H-3A, H-3B); ${ }^{13}$ C-NMR: 13.4 (C-12B), 13.6 (C-12A), 14.3 (C-13A), 14.3 (C-13B), 25.1 (C-9A), 25.1 (C-9B), 29.5 (C-5B), 29.5 (C-5A), 32.1 (C-10A), 32.2 (C-10B), 36.6 (C-7B), 36.7 (C-7A), 38.5 (C-4B), 38.89 (C-4A), 39.1 (C-6B), 40.1 (C-6A), 43.8 (C-1A), 44.1 (C-1B), 60.1 (C-12A), 60.1 (C-12B), 126.7 (C-3A), $127.2(\mathrm{C}-3 \mathrm{~B}), 127.5$ (C-2A), 128.0 (C-2B), 176.0 (C-8B), 176.1 (C-8A); IR (KBr, cm $\left.{ }^{-1}\right)$ : 2952, 1732, 1464, 1365, 1180; ESIHRMS: calcd. for $\mathrm{C}_{13} \mathrm{H}_{22} \mathrm{O}_{2}, m / z 211.1698[\mathrm{M}+\mathrm{H}]^{+}$, found 211.1691. (Supplementary Materials, Figures S1-S6).

2b: $\mathrm{n}_{\mathrm{D}}=1.4645 ;{ }^{1} \mathrm{H}-\mathrm{NMR}: 0.98$ (s, 9H, $\left.\mathrm{CH}_{3}-9 \mathrm{~A}, \mathrm{CH}_{3}-10 \mathrm{~A}, \mathrm{CH}_{3}-10 \mathrm{~B}\right), 0.99$ (s, 3H, $\left.\mathrm{CH}_{3}-9 \mathrm{~B}\right), 1.08$ (d, $\left.J=7.2 \mathrm{~Hz}, 3 \mathrm{H}, \mathrm{CH}_{3}-12 \mathrm{~A}\right), 1.15\left(\mathrm{~d}, J=7.2 \mathrm{~Hz}, 3 \mathrm{H}, \mathrm{CH}_{3}-12 \mathrm{~B}\right), 1.11\left(\mathrm{~s}, 3 \mathrm{H}, \mathrm{CH}_{3}-12 \mathrm{~A}\right), 1.14\left(\mathrm{~s}, 3 \mathrm{H}, \mathrm{CH}_{3}-11 \mathrm{~B}\right)$, $1.26\left(\mathrm{t}, J=7.2 \mathrm{~Hz}, 3 \mathrm{H}, \mathrm{CH}_{3}-14 \mathrm{~A}\right), 1.28\left(\mathrm{t}, J=7.2 \mathrm{~Hz}, 3 \mathrm{H}, \mathrm{CH}_{3}-14 \mathrm{~B}\right), 1.63-1.80\left(\mathrm{~m}, 8 \mathrm{H}, \mathrm{CH}_{2}-4 \mathrm{~A}, \mathrm{CH}_{2}-4 \mathrm{~B}\right.$, $\left.\mathrm{CH}_{2}-6 \mathrm{~A}, \mathrm{CH}_{2}-6 \mathrm{~A}\right), 2.32(\mathrm{q}, J=7.2 \mathrm{~Hz}, 1 \mathrm{H}, \mathrm{H}-7 \mathrm{~A}), 2.33(\mathrm{q}, J=7.2 \mathrm{~Hz}, 1 \mathrm{H}, \mathrm{H}-7 \mathrm{~B}), 4.07-4.12(\mathrm{~m}, 2 \mathrm{H}$, $\left.\mathrm{CH}_{2}-13 \mathrm{~A}\right), 4.13-4.16\left(\mathrm{~m}, 2 \mathrm{H}, \mathrm{CH}_{2}-13 \mathrm{~B}\right), 5.30(\mathrm{~d}, J=10.2 \mathrm{~Hz}, 1 \mathrm{H}, \mathrm{H}-2 \mathrm{~B}), 5.57(\mathrm{~d}, J=10.2 \mathrm{~Hz}, 1 \mathrm{H}, \mathrm{H}-2 \mathrm{~A})$, $5.64(\mathrm{ddd}, J=10.2,5.4$ and $2.4 \mathrm{~Hz}, 1 \mathrm{H}, \mathrm{H}-3 \mathrm{~A}), 5.68(\mathrm{ddd}, J=10.2,6.0$ and $3.0 \mathrm{~Hz}, 1 \mathrm{H}, \mathrm{H}-3 \mathrm{~B}) ;{ }^{13} \mathrm{C}-\mathrm{NMR}$ : 12.1 (C-12A), 12.6 (C-12B), 14.3 (C-14A), 14.4 (C-14B), 25.5 (C-11A), 25.9 (C-11B), 28.1 (C-10B), 28.2 (C-10A), 29. 9 (C-1B), 29.0 (C-1A), 32.6 (C-9A), 32.6 (C-9B), 37.6 (C-5A), 38.0 (C-5B), 38.3 (C-4B), 38.4 (C-6B), 43.7 (C-4A), 44.4 (C-6A), 50.2 (C-7B), 50.3 (C-7A), 59.8 (C-13A), 59.9 (C-13B), 125.1 (C-3A), 125.4 (C-3B), 132.7 (C-2A), 133.5 (C-2B), 175.5 (C-8A), 175.6 (C-8B); IR (KBr, cm $\left.{ }^{-1}\right)$ : 2953, 1732, 1457, 1369, 1175; ESIHRMS: calcd. for $\mathrm{C}_{14} \mathrm{H}_{24} \mathrm{O}_{2}, m / z 225.1855[\mathrm{M}+\mathrm{H}]^{+}$, found 225.1860. (Supplementary Materials, Figures S47-S52).

3.2.2. (5,5,7-Trimethylcyclohex-2-en-1-yl)acetic acid (3a) and (1,5,5,7-Tetramethylcyclohex-2-en1 -yl)acetic acid (3b)

Basic hydrolysis of the mixtures of two diastereoisomers of esters $\mathbf{3 a}$ and $\mathbf{3} \mathbf{b}$ (according to a previously described procedure [28] gave $3.5 \mathrm{~g}(19.2 \mathrm{mmol}$, yield $96 \%)$ of acid 3a (two diastereoisomers and $3.6 \mathrm{~g}$ (18.4 mmol, yield $92 \%$ ) of acid $3 \mathbf{b}$ (two diastereoisomers with the following physical and spectral data:

3a: $\mathrm{n}_{\mathrm{D}}=1.4719 ;{ }^{1} \mathrm{H}-\mathrm{NMR}: 1.00\left(\mathrm{~s}, 9 \mathrm{H}, \mathrm{CH}_{3}-9 \mathrm{~B}, \mathrm{CH}_{3}-10 \mathrm{~A}, \mathrm{CH}_{3}-10 \mathrm{~B}\right), 1.01$ (s, 3H, $\left.\mathrm{CH}_{3}-9 \mathrm{~A}\right), 1.12$ (d, $\left.J=7.5 \mathrm{~Hz}, 3 \mathrm{H}, \mathrm{CH}_{3}-11 \mathrm{~A}\right), 1.16\left(\mathrm{~s}, 2 \mathrm{H}, \mathrm{CH}_{2}-4 \mathrm{~B}\right), 1.16(\mathrm{~s}, 1 \mathrm{H}, \mathrm{H}-1 \mathrm{~A}), 1.17(\mathrm{~s}, 1 \mathrm{H}, \mathrm{H}-1 \mathrm{~B}), 1.20(\mathrm{~s}, 3 \mathrm{H}$, $\left.\mathrm{CH}_{3}-11 \mathrm{~B}\right), 1.63\left(\mathrm{~d}, J=14.0 \mathrm{~Hz}, 2 \mathrm{H}, \mathrm{CH}_{2}-4 \mathrm{~B}\right), 1.74-1.75\left(\mathrm{~m}, 2 \mathrm{H}, \mathrm{CH}_{2}-6 \mathrm{~A}\right), 1.77-1.79\left(\mathrm{~m}, 2 \mathrm{H}, \mathrm{CH}_{2}-4 \mathrm{~B}\right)$, 2.35-2.38 (m, 2H, $\left.\mathrm{CH}_{2}-7 \mathrm{~A}, \mathrm{CH}_{2}-7 \mathrm{~B}\right), 5.34-5.36(\mathrm{~m}, 1 \mathrm{H}, \mathrm{H}-2 \mathrm{~B}), 5.59-5.61(\mathrm{~m}, 1 \mathrm{H}, \mathrm{H}-2 \mathrm{~A}), 5.68-5.73$ (m, 2H, H-3A, H-3B); ${ }^{13} \mathrm{C}-\mathrm{NMR}: 12.2$ (C-11A), 12.6 (C-11B), 25.8 (C-1A), 25.9 (C-1B), 28.2 (C-9A), 28.2 (C-9B), 29.9 (C-5B), 30.0 (C-5A), 32.5 (C-10A), 32.6 (C-10B), 37.9 (C-6A), 38.3 (C-6B), 43.5 (C-4B), 44.7 (C-4A), 50.1 (C-7B), 50.2 (C-7A), 125.6 (C-3A), 125.8 (C-3B), 132.2 (C-2A), 133.2 (C-2B), 181.6 (C-8B), 181.6 (C-8A); IR (KBr, cm ${ }^{-1}$ ): 2952, 1706, 1457, 1365, 1206; ESIHRMS: calcd. for $\mathrm{C}_{11} \mathrm{H}_{18} \mathrm{O}_{2} \mathrm{Na}, \mathrm{m} / \mathrm{z}$ $205.1205[\mathrm{M}+\mathrm{H}]^{+}$, found 205.1210. (Supplementary Materials, Figures S7-S12).

3b: m.p. $=43-45^{\circ} \mathrm{C} ;{ }^{1} \mathrm{H}-\mathrm{NMR}: 0.99$ (s, 9H, $\left.\mathrm{CH}_{3}-9 \mathrm{~A}, \mathrm{CH}_{3}-10 \mathrm{~A}, \mathrm{CH}_{3}-10 \mathrm{~B}\right), 1.00$ (s, 3H, $\left.\mathrm{CH}_{3}-9 \mathrm{~B}\right), 1.12(\mathrm{~d}$, $\left.J=7.2 \mathrm{~Hz}, 3 \mathrm{H}, \mathrm{CH}_{3}-12 \mathrm{~B}\right), 1.51\left(\mathrm{~s}, 3 \mathrm{H}, \mathrm{CH}_{3}-11 \mathrm{~A}\right), 1.62\left(\mathrm{t}, J=7.2 \mathrm{~Hz}, 3 \mathrm{H}, \mathrm{CH}_{3}-12 \mathrm{~A}\right), 1.20\left(\mathrm{~s}, 3 \mathrm{H}, \mathrm{CH}_{3}-11 \mathrm{~B}\right)$, $1.62\left(\mathrm{~d}, J=13.8 \mathrm{~Hz}, 2 \mathrm{H}, \mathrm{CH}_{2}-6 \mathrm{~B}\right), 1.67-1.75\left(\mathrm{~m}, 4 \mathrm{H}, \mathrm{CH}_{2}-4 \mathrm{~A}, \mathrm{CH}_{2}-4 \mathrm{~B}\right), 1.77-1.81\left(\mathrm{~m}, 2 \mathrm{H}, \mathrm{CH}_{2}-6 \mathrm{~A}\right)$, $2.36(\mathrm{q}, J=7.2 \mathrm{~Hz}, 1 \mathrm{H}, \mathrm{H}-7 \mathrm{~B}), 2.37(\mathrm{q}, J=6.6 \mathrm{~Hz}, 1 \mathrm{H}, \mathrm{H}-7 \mathrm{~A}), 5.60(\mathrm{~d}, J=10.2 \mathrm{~Hz}, 1 \mathrm{H}, \mathrm{H}-2 \mathrm{~A}), 5.57(\mathrm{~d}$, $J=10.2 \mathrm{~Hz}, 1 \mathrm{H}, \mathrm{H}-2 \mathrm{~B}), 5.64(\mathrm{ddd}, J=10.2,5.4$ and $2.4 \mathrm{~Hz}, 1 \mathrm{H}, \mathrm{H}-3 \mathrm{~A}), 5.68(\mathrm{ddd}, J=10.2,5.4$ and $2.4 \mathrm{~Hz}$, 1H, H-3B); ${ }^{13}$ C-NMR: 12.2 (C-12A), 12.6 (C-12B), 25.3 (C-11A), 25.9 (C-11B), 28.2 (C-10A), 28.2 (C-10B), 29.9 (C-1B), 29.0 (C-1A), 32.5 (C-9A), 32.6 (C-9B), 37.8 (C-5B), 38.0 (C-5A), 38.3 (C-4A), 38.3 (C-4B), 43.5 (C-6B), 44.8 (C-6A), 50.2 (C-7B), 50.3 (C-7A), 125.6 (C-3A), 125.8 (C-3B), 132.2 (C-2A), 133.3 (C-2B), 181.8 (C-8B), 181.9 (C-8A); IR ( $\left.\mathrm{KBr}, \mathrm{cm}^{-1}\right)$ : 2955, 1703, 1460, 1248, 1074; ESIHRMS: calcd. for $\mathrm{C}_{12} \mathrm{H}_{20} \mathrm{O}_{2}, \mathrm{~m} / \mathrm{z}$ 197.1542 [M + H $]^{+}$, found 197.1532. (Supplementary Materials, Figures S53-S58). 
3.2.3. 2-Iodo-4,4,7-trimethyl-9-oxabicyclo[4.3.0]nonan-8-one (4a) and 2-Iodo-4,4,6,7-tetramethyl-9oxabicyclo-[4.3.0]nonan-8-one (4b)

According to the known procedure [28] we obtained $4.2 \mathrm{~g}, 13.6 \mathrm{mmol}$ (yield 71\%) of iodolactone $\mathbf{4 a}$ as a mixture of diastereoisomers and $4.4 \mathrm{~g}, 13.7 \mathrm{mmol}$ (yield $75 \%$ ) and $\mathbf{4 b}$ characterised by the data presented below:

4a: m.p. $=108-110{ }^{\circ} \mathrm{C} ;{ }^{1} \mathrm{H}-\mathrm{NMR}: 0.99$ (s, 3H, $\left.\mathrm{CH}_{3}-9 \mathrm{~A}\right), 1.01$ (s, 3H, $\left.\mathrm{CH}_{3}-9 \mathrm{~B}\right), 1.03$ (s, 3H, $\left.\mathrm{CH}_{3}-10 \mathrm{~A}\right)$, $1.09\left(\mathrm{~d}, J=7.2 \mathrm{~Hz}, 3 \mathrm{H}, \mathrm{CH}_{3}-11 \mathrm{~A}\right), 1.10$ (s, 3H, $\left.\mathrm{CH}_{3}-10 \mathrm{~B}\right), 1.11\left(\mathrm{~d}, J=7.2 \mathrm{~Hz}, 3 \mathrm{H}, \mathrm{CH}_{3}-11 \mathrm{~B}\right), 1.23(\mathrm{~s}, 2 \mathrm{H}$, $\left.\mathrm{CH}_{2}-5\right), 1.35\left(\mathrm{~d}, \mathrm{~J}=15.2 \mathrm{~Hz}, 1 \mathrm{H}\right.$, one of $\left.\mathrm{CH}_{2}-3 \mathrm{~A}\right), 1.48\left(\mathrm{~s}, 2 \mathrm{H}, \mathrm{CH}_{2}-5 \mathrm{~B}\right), 1.75(\mathrm{dd}, \mathrm{J}=15.2$ and $2.5 \mathrm{~Hz}, 1 \mathrm{H}$, one of $\left.\mathrm{CH}_{2}-3 \mathrm{~A}\right), 1.99(\mathrm{dd}, J=14.8$ and $4.6 \mathrm{~Hz}, 1 \mathrm{H}, \mathrm{H}-6 \mathrm{~B}), 2.02\left(\mathrm{t}, J=13.7 \mathrm{~Hz}, 1 \mathrm{H}\right.$, one of $\left.\mathrm{CH}_{2}-3 \mathrm{~B}\right), 2.10$ $(\mathrm{dd}, J=14.8$ and $9.9 \mathrm{~Hz}, 1 \mathrm{H}, \mathrm{H}-6 \mathrm{~A}), 2.19-2.22\left(\mathrm{~m}, 1 \mathrm{H}\right.$, one of $\left.\mathrm{CH}_{2}-3 \mathrm{~B}\right), 2.42(\mathrm{q}, J=7.2 \mathrm{~Hz}, 1 \mathrm{H}, \mathrm{H}-7 \mathrm{~A})$, $2.92(\mathrm{q}, J=7.1 \mathrm{~Hz}, 1 \mathrm{H}, \mathrm{H}-7 \mathrm{~B}), 4.23(\mathrm{ddd}, J=17.5,13.8$ and $3.8 \mathrm{~Hz}, 1 \mathrm{H}, \mathrm{H}-2 \mathrm{~B}), 4.30-4.33(\mathrm{~m}, 2 \mathrm{H}, \mathrm{H}-2 \mathrm{~A}$, H-1B), 4.68 (d, J = 5.0 Hz, 1H, H-1A); ${ }^{13} \mathrm{C}-\mathrm{NMR}: 7.6$ (C-11B), 7.6 (C-11A), 20.4 (C-2A), 25.7 (C-10A), 25.8 (C-10B), 26.0 (C-5A), 26.2 (C-2B), 30.0 (C-5B), 33.7 (C-9A), 34.0 (C-9B), 38.1 (C-6B), 40.5 (C-7B), 43.0 (C-6A), 43.1 (C-4A), 43.4 (C-4B), 44.2 (C-6A), 49.4 (C-3B), 49.6 (C-7A), 89.8 (C-1A), 91.1 (C-1B), 177.0 (C-8A), 177.5 (C-8B); IR (KBr, cm $\left.{ }^{-1}\right)$ : 2955, 1779, 1464, 1164, 1024; ESIHRMS: calcd. for $\mathrm{C}_{11} \mathrm{H}_{17} \mathrm{IO}_{2}, \mathrm{~m} / z$ 309.0346 [M $+\mathrm{H}]^{+}$, found 309.0352. (Supplementary Materials, Figures S13-S18).

4b-A: m.p. $=110-112{ }^{\circ} \mathrm{C} ;{ }^{1} \mathrm{H}-\mathrm{NMR}: 0.99$ (s, 3H, $\left.\mathrm{CH}_{3}-9\right), 1.10$ (d, J = 7.1 Hz, 3H, CH -12$), 1.11$ (s, 3H, $\left.\mathrm{CH}_{3}-11\right), 1.12\left(\mathrm{~d}, J=7.1 \mathrm{~Hz}, 3 \mathrm{H}, \mathrm{CH}_{3}-12\right), 1.11-1.26\left(\mathrm{~m}, 1 \mathrm{H}\right.$, one of $\left.\mathrm{CH}_{2}-5\right), 1.19-1.21(\mathrm{~m}, 1 \mathrm{H}$, one of $\left.\mathrm{CH}_{2}-5\right), 1.49\left(\mathrm{~s}, 3 \mathrm{H}, \mathrm{CH}_{2}-12\right), 1.99\left(\mathrm{dd}, J=14.7\right.$ and $4.5 \mathrm{~Hz}, 1 \mathrm{H}$, one of $\left.\mathrm{CH}_{2}-3\right), 2.11(\mathrm{dd}, J=14.7$ and $9.7 \mathrm{~Hz}, 1 \mathrm{H}$, one of $\left.\mathrm{CH}_{2}-3\right), 2.42(\mathrm{q}, J=7.2 \mathrm{~Hz}, 1 \mathrm{H}, \mathrm{H}-7), 4.32(\mathrm{~m}, 1 \mathrm{H}, \mathrm{H}-2), 4.33(\mathrm{~d}, J=5.0 \mathrm{~Hz}$, 1H, H-1); ${ }^{13}$ C-NMR: 7.6 (C-11), 20.4 (C-2), 26.0 (C-12), 29.7 (C-4), 30.0 (C-10), 31.4 (C-6), 33.7 (C-9), 38.1 (C-5), 43.0 (C-3), 49.7 (C-7), 89.8 (C-1), 177.0 (C-8); IR (KBr, cm ${ }^{-1}$ ): 2956, 1763, 1465, 1189, 1009; ESIHRMS: calcd. for $\mathrm{C}_{12} \mathrm{H}_{19} \mathrm{IO}_{2} \mathrm{Na}, m / z$ 345.0327 $[\mathrm{M}+\mathrm{H}]^{+}$, found 345.0322. (Supplementary Materials, Figures S59-S64).

4b-B: m.p. $=137-139{ }^{\circ} \mathrm{C} ;{ }^{1} \mathrm{H}-\mathrm{NMR}: 1.01$ (s, 3H, $\left.\mathrm{CH}_{3}-9\right), 1.03$ (s, 3H, $\left.\mathrm{CH}_{3}-10\right), 1.11$ (s, 3H, $\mathrm{CH}_{3}-11$ ), $1.12\left(\mathrm{~d}, J=7.1 \mathrm{~Hz}, 3 \mathrm{H}, \mathrm{CH}_{3}-12\right), 1.36\left(\mathrm{~d}, J=15.2 \mathrm{~Hz}, 1 \mathrm{H}\right.$, one of $\left.\mathrm{CH}_{2}-5\right), 1.77(\mathrm{dd}, J=15.2$ and $2.5 \mathrm{~Hz}$, $1 \mathrm{H}$, one of $\left.\mathrm{CH}_{2}-5\right), 2.03\left(\mathrm{t}, J=13.7 \mathrm{~Hz}, 1 \mathrm{H}\right.$, one of $\left.\mathrm{CH}_{2}-3\right), 2.21(\mathrm{dd}, J=13.7$ and $2.7 \mathrm{~Hz}, 1 \mathrm{H}$, one of $\left.\mathrm{CH}_{2}-3\right), 2.93(\mathrm{q}, J=7.1 \mathrm{~Hz}, 1 \mathrm{H}, \mathrm{H}-7), 4.24(\mathrm{ddd}, J=13.9,10.2$ and $3.8 \mathrm{~Hz}, 1 \mathrm{H}, \mathrm{H}-2), 4.33(\mathrm{~d}, J=10.2 \mathrm{~Hz}$, 1H, H-1); ${ }^{13} \mathrm{C}-\mathrm{NMR}: 7.6$ (C-11), 25.6 (C-9), 25.78 (C-12), 26.2 (C-2), 34.0 (C-10), 34.1 (C-4), 40.6 (C-7), 43.4 (C-6), 44.3 (C-5), 49.4 (C-3), 91.1 (C-1), 177.5 (C-8); IR (KBr, cm ${ }^{-1}$ ): 2937, 1779, 1456, 1163, 1024; ESIHRMS: calcd. for $\mathrm{C}_{12} \mathrm{H}_{19} \mathrm{IO}_{2} \mathrm{Na}, m / z$ 345.0327 [M+H] $]^{+}$, found 345.0323. (Supplementary Materials, Figures S65-S70).

3.2.4. 4,4,7-Trimethyl-9-oxabicyclo[4.3.0]non-2-en-8-one (5a) and 4,4,6,7-Trimethyl-9-oxabicyclo [4.3.0]non-2-en-8-one (5b)

Dehydrodehalogenation of this mixture, according to the known procedure [28], gave as a diastereoisomeric mixture $1.9 \mathrm{~g}, 10.6 \mathrm{mmol}$ (yield 79\%) of unsaturated lactone 5a and $2.1 \mathrm{~g}, 10.8 \mathrm{mmol}$ (yield $79 \%$ ) of $5 \mathrm{~b}$ unsaturated lactone with the following physical and spectral data:

5a: m.p. $=74-76{ }^{\circ} \mathrm{C} ;{ }^{1} \mathrm{H}-\mathrm{NMR}: 1.02$ (s, 6H, $\left.\mathrm{CH}_{3}-9 \mathrm{~A}, \mathrm{CH}_{3}-9 \mathrm{~B}\right), 1.07$ (s, 6H, CH $\left.3-10 \mathrm{~A}, \mathrm{CH}_{3}-10 \mathrm{~B}\right), 1.08-1.14$ $\left(\mathrm{m}, 1 \mathrm{H}\right.$, one of $\left.\mathrm{CH}_{2}-5 \mathrm{~B}\right), 1.20\left(\mathrm{~d}, \mathrm{~J}=7.2 \mathrm{~Hz}, 3 \mathrm{H}, \mathrm{CH}_{3}-11 \mathrm{~B}\right), 1.30-1.34\left(\mathrm{~m}, 1 \mathrm{H}\right.$, one of $\left.\mathrm{CH}_{2}-5 \mathrm{~A}\right), 1.37(\mathrm{~d}$, $\left.J=7.8 \mathrm{~Hz}, 3 \mathrm{H}, \mathrm{CH}_{3}-11 \mathrm{~A}\right), 1.45\left(\mathrm{dd}, J=12.0\right.$ and $3.6 \mathrm{~Hz}, 1 \mathrm{H}$, one of $\left.\mathrm{CH}_{2}-5 \mathrm{~B}\right), 1.55(\mathrm{dd}, J=13.8$ and $4.2 \mathrm{~Hz}, 1 \mathrm{H}$, one of $\left.\mathrm{CH}_{2}-5 \mathrm{~A}\right), 2.28-2.32(\mathrm{~m}, 1 \mathrm{H}, \mathrm{H}-6 \mathrm{~A}), 2.41$ (q, J = 7.6 Hz, 1H, H-7A), 2.62-2.68 (m, $1 \mathrm{H}$, H-6B), 2.96 (quintet, $J=7.3 \mathrm{~Hz}, 1 \mathrm{H}, \mathrm{H}-7 \mathrm{~B}), 4.62(\mathrm{t}, J=4.7 \mathrm{~Hz}, 1 \mathrm{H}, \mathrm{H}-1 \mathrm{~B}), 4.81(\mathrm{t}, J=4.9 \mathrm{~Hz}, 1 \mathrm{H}, \mathrm{H}-1 \mathrm{~A})$, 5.78-5.81 (m, 2H, H-2A, H-2B), 5.90-5.93 (m, 2H, H-3A, H-3B); ${ }^{13} \mathrm{C}-\mathrm{NMR}: 9.2$ (C-11B), 15.5 (C-11A), 26.8 (C-9B), 27.2 (C-9A), 30.1 (C-10A), 30.5 (C-10B), 31.7 (C-4A), 31.8 (C-4B), 33.5 (C-5B), 35.3 (C-5A), 38.4 (C-6B), 38.6 (C-6A), 40.0 (C-7B), 43.2 (C-7A), 73.1 (C-1B), 73.2 (C-1A), 119.7 (C-2B), 119.8 (C-2A), 144.8 (C-3A), 145.3 (C-3B), 178.8 (C-8A), 178.89 (C-8B); IR (KBr, cm $\left.{ }^{-1}\right):$ 2960, 1768, 1470, 1381, 1172, 967; 
ESIHRMS: calcd. for $\mathrm{C}_{11} \mathrm{H}_{16} \mathrm{O}_{2}, m / z 181.1228[\mathrm{M}+\mathrm{H}]^{+}$, found 181.1221. (Supplementary Materials, Figures S19-S24).

5b-A: $\mathrm{n}_{\mathrm{D}}=1.4810 ;{ }^{1} \mathrm{H}-\mathrm{NMR}: 1.02\left(\mathrm{~s}, 3 \mathrm{H}, \mathrm{CH}_{3}-9\right), 1.06\left(\mathrm{~s}, 3 \mathrm{H}, \mathrm{CH}_{3}-10\right), 1.09$ (s, 3H, $\left.\mathrm{CH}_{3}-12\right), 1.10$ (d, $\left.J=7.3 \mathrm{~Hz}, 3 \mathrm{H}, \mathrm{CH}_{3}-11\right), 1.47\left(\mathrm{~d}, J=14.9 \mathrm{~Hz}, 1 \mathrm{H}\right.$, one of $\left.\mathrm{CH}_{2}-5\right), 1.71(\mathrm{dd}, J=14.9$ and $2.5 \mathrm{~Hz}, 1 \mathrm{H}$, one of $\left.\mathrm{CH}_{2}-5\right), 2.03\left(\mathrm{t}, J=13.7 \mathrm{~Hz}, 1 \mathrm{H}\right.$, one of $\left.\mathrm{CH}_{2}-3\right), 2.21\left(\mathrm{dd}, J=13.7\right.$ and $2.7 \mathrm{~Hz}, 1 \mathrm{H}$, one of $\left.\mathrm{CH}_{2}-3\right), 2.78$ (q, $J=7.3 \mathrm{~Hz}, 1 \mathrm{H}, \mathrm{H}-7), 4.49(\mathrm{~s}, 1 \mathrm{H}, \mathrm{H}-1), 5.56(\mathrm{dd}, J=10.2$ and $2.5 \mathrm{~Hz}, 1 \mathrm{H}, \mathrm{H}-2), 5.69(\mathrm{~d}, J=10.2 \mathrm{~Hz}$, 1H, H-3); ${ }^{13}$ C-NMR: 7.6 (C-11), 22.9 (C-9), 29.4 (C-12), 31.5 (C-6), 33.0 (C-10), 40.4 (C-4), 41.1 (C-7), 42.3 (C-5), 81.0 (C-1), 120.6 (C-2), 140.6 (C-3), 178.9 (C-8); IR (KBr, cm ${ }^{-1}$ ): 2962, 1775, 1456, 1378, 1169, 977, ESIHRMS: calcd. for $\mathrm{C}_{12} \mathrm{H}_{18} \mathrm{O}_{2}, \mathrm{~m} / z$ 195.1385 $[\mathrm{M}+\mathrm{H}]^{+}$, found 195.1391. (Supplementary Materials, Figures S71-S76).

5b-B: $\mathrm{n}_{\mathrm{D}}=1.4810 ;{ }^{1} \mathrm{H}-\mathrm{NMR}: 1.04\left(\mathrm{~s}, 3 \mathrm{H}, \mathrm{CH}_{3}-9\right), 1.12\left(\mathrm{~s}, 3 \mathrm{H}, \mathrm{CH}_{3}-10\right), 1.13\left(\mathrm{~d}, J=7.2 \mathrm{~Hz}, 3 \mathrm{H}, \mathrm{CH}_{3}-11\right)$, $1.24\left(\mathrm{~s}, 3 \mathrm{H}, \mathrm{CH}_{3}-12\right), 1.28\left(\mathrm{~d}, J=14.0 \mathrm{~Hz}, 1 \mathrm{H}\right.$, one of $\left.\mathrm{CH}_{2}-5\right), 1.36(\mathrm{dd}, J=14.0$ and $2.5 \mathrm{~Hz}, 1 \mathrm{H}$, one of $\left.\mathrm{CH}_{2}-5\right) 2.44(\mathrm{q}, J=7.2 \mathrm{~Hz}, 1 \mathrm{H}, \mathrm{H}-7), 4.28(\mathrm{~d}, J=5.2 \mathrm{~Hz}, 1 \mathrm{H}, \mathrm{H}-1), 5.77(\mathrm{dd}, J=10.0$ and $5.2 \mathrm{~Hz}, 1 \mathrm{H}$, $\mathrm{H}-2), 5.89(\mathrm{~d}, J=10.0 \mathrm{~Hz}, 1 \mathrm{H}, \mathrm{H}-3) ;{ }^{13} \mathrm{C}-\mathrm{NMR}: 7.2$ (C-11), 23.0 (C-12), 28.4 (C-10), 31.8 (C-6), 33.1 (C-9), 38.3 (C-5), 40.2 (C-4), 49.9 (C-7), 78.3 (C-1), 117.6 (C-2), 144.7 (C-3), 178.5 (C-8); IR (KBr, cm ${ }^{-1}$ ): 2961, 1771, 1457, 1379, 1169, 976; ESIHRMS: calcd. for $\mathrm{C}_{12} \mathrm{H}_{18} \mathrm{O}_{2}, m / z 195.1385[\mathrm{M}+\mathrm{H}]^{+}$, found 195.1322 . (Supplementary Materials, Figures S77-S82).

\subsection{Biotransformations}

\subsubsection{Microorganisms}

The fungal and yeast strains which were used for biotransformation came from the collection of the Department of Chemistry, Wrocław University of Environmental and Life Sciences (Wrocław, Poland). The following strains were used: Fusarium culmorum AM10, Fusarium avenaceum AM11, Fusarium oxysporum AM13, Fusarium tricinctum AM16, Fusarium semitectum AM20, Penicilium vermiculatum AM30, Penicillium albidum AM79, Penicillium camembertii AM83, Penicilium chermesinum AM113, Absidia corerulea AM93, Absidia glauca AM254, Absidia glauca AM177, Absidia cylindrospora AM336, Syncephalastrum racemosum AM105. All of these strains were cultivated on Sabouraud's agar consisting of aminobac $(0.5 \%)$, peptone $(0.5 \%)$, glucose $(4 \%)$ and agar $(1.5 \%)$ dissolved in distilled water at $28{ }^{\circ} \mathrm{C}$ and stored in a refrigerator at $4{ }^{\circ} \mathrm{C}$.

\subsubsection{Screening Procedure}

Each strain of the fungus was cultured in two $300 \mathrm{~mL}$ Erlenmeyer flasks containing standard medium ( $3 \mathrm{~g}$ of glucose, $1 \mathrm{~g}$ of peptone, dissolved in $100 \mathrm{~mL}$ of distilled water). After three days $10 \mathrm{mg}$ of substrate dissolved in $1 \mathrm{~mL}$ of acetone was added to each flask with the grown culture. Incubation of the shaken cultures with substrate was continued. After 7 and 14 days of incubation, the medium were extracted with dichloromethane $(15 \mathrm{~mL})$ and analyzed by GC (DB-17) column.

\subsubsection{Preparative Biotransformation}

Preparative biotransformation was carried out in ten $300 \mathrm{~mL}$ Erlenmeyer flasks containing cultures of 3-day fungal strains (prepared in a similar manner as described in the screening procedure). Substrate $(100 \mathrm{mg})$ was dissolved in $10 \mathrm{~mL}$ of acetone and added to ten bottles. After 14 days the reaction mixture was extracted with dichloromethane $(3 \times 40 \mathrm{~mL})$. The combined organic fractions were dried $\left(\mathrm{MgSO}_{4}\right)$ and evaporated under reduced pressure. The pure product was purified by column chromatography (silica gel, hexane-acetone 3:1). As a result of these reactions two products were obtained.

1-Hydroxy-4,4,7-trimethyl-9-oxabicyclo[4.3.0]non-2-en-8-one (6a) was characterized by the following physical and spectral properties: m.p. $=77-78{ }^{\circ} \mathrm{C} ;{ }^{1} \mathrm{H}-\mathrm{NMR}: 1.14\left(\mathrm{~d}, J=7.2 \mathrm{~Hz}, 3 \mathrm{H}, \mathrm{CH}_{3}-11 \mathrm{~A}\right)$, 1.19 (s, 3H, $\left.\mathrm{CH}_{3}-9 \mathrm{~A}\right), 1.20$ (s, 3H, $\left.\mathrm{CH}_{3}-9 \mathrm{~B}\right), 1.23$ (s, 3H, $\left.\mathrm{CH}_{3}-10 \mathrm{~B}\right), 1.24$ (s, 3H, $\left.\mathrm{CH}_{3}-10 \mathrm{~A}\right), 1.25$ (d, 
$\left.J=7.2 \mathrm{~Hz}, 3 \mathrm{H}, \mathrm{CH}_{3}-11 \mathrm{~B}\right), 1.78-1.79\left(\mathrm{~m}, 2 \mathrm{H}, \mathrm{CH}_{2}-5 \mathrm{~A}\right), 1.80-1.81$ (m, $\left.1 \mathrm{H}, \mathrm{CH}_{2}-5 \mathrm{~B}\right), 1.95-2.00(\mathrm{~m}, 1 \mathrm{H}$, one of $\left.\mathrm{CH}_{2}-5 \mathrm{~B}\right), 2.91-2.95(\mathrm{~m}, 1 \mathrm{H}$, one of H-6B), 3.02-3.04 (m, 1H, H-7B), 3.06-3.09 (m, 1H, H-6A), 3.16-3.18 $(\mathrm{m}, 1 \mathrm{H}, \mathrm{H}-7 \mathrm{~A}), 5.86(\mathrm{~d}, J=10.0 \mathrm{~Hz}, 1 \mathrm{H}, \mathrm{H}-3 \mathrm{~A}), 5.87$ (d, J = 10.0 Hz, 1H, H-3B), 6.63-6.66 (m, 2H, H-2A, H-2B); ${ }^{13}$ C-NMR: 12.0 (C-11A), 12.2 (C-11B), 24.3 (C-10A), 24.8 (C-10B), 30.1 (C-9B), 30.2 (C-9A), 33.2 (C-4A), 33.2 (C-4B), 37.4 (C-7B), 37.4 (C-7A), 38.2 (C-5B), 38.3 (C-5A), 44.3 (C-6A), 44.7 (C-6B), 125.9 (C-3A), 126.1 (C-3B), 158.6 (C-2B), 158.7 (C-2A), 179.4 (C-1B), 181.3 (C-1A), 198.6 (C-8A), 198.7 (C-8B); ESIHRMS: calcd. for $\mathrm{C}_{11} \mathrm{H}_{16} \mathrm{O}_{3}, m / z$ 197.1178 [M+ H] $]^{+}$, found 197.1173. (Supplementary Materials, Figures S25-S29).

6-Hydroxy-4,4,7-trimethyl-9-oxabicyclo[4.3.0]non-2-en-8-one (7a) was characterized by the following physical and spectral properties: m.p. $=76-77{ }^{\circ} \mathrm{C} ;{ }^{1} \mathrm{H}-\mathrm{NMR}: 1.04\left(\mathrm{~s}, 3 \mathrm{H}, \mathrm{CH}_{3}-9\right), 1.09\left(\mathrm{~s}, 3 \mathrm{H}, \mathrm{CH}_{3}-10\right)$, $1.43\left(\mathrm{~d}, J=5.0 \mathrm{~Hz}, 3 \mathrm{H}, \mathrm{CH}_{3}-11\right), 1.45\left(\mathrm{dd}, J=14.9\right.$ and $\left.4.5 \mathrm{~Hz}, 2 \mathrm{H}, \mathrm{CH}_{2}-5\right), 2.50(\mathrm{dt}, J=14.4$ and $4.8 \mathrm{~Hz}$, $1 \mathrm{H}, \mathrm{H}-7), 5.03(\mathrm{dd}, J=8.8$ and $4.4 \mathrm{~Hz}, 1 \mathrm{H}, \mathrm{H}-1), 5.83(\mathrm{dd}, J=9.9$ and $4.4 \mathrm{~Hz}, 1 \mathrm{H}, \mathrm{H}-2), 5.94(\mathrm{~d}, J=9.9 \mathrm{~Hz}$, 1H, H-3); ${ }^{13}$ C-NMR: 19.1 (C-11), 26.7 (C-9), 30.3 (C-10), 32.1 (C-4), 34.0 (C-5), 42.0 (C-7), 73.0 (C-1), 77.7 (C-6), 119.6 (C-2), 145.1 (C-3), 177.1 (C-8); ESIHRMS: calcd. for $\mathrm{C}_{11} \mathrm{H}_{16} \mathrm{O}_{3}, m / z 197.1178$ [M + H] , found 197.1176. (Supplementary Materials, Figures S30-S34).

10-Hydroxy-4,4,7-trimethyl-9-oxabicyclo[4.3.0]non-2-en-8-one (8a) was characterized by the following physical and spectral properties: colourless oil; ${ }^{1} \mathrm{H}-\mathrm{NMR}: 1.06\left(\mathrm{~s}, 3 \mathrm{H}, \mathrm{CH}_{3}-9\right), 1.20-1.22(\mathrm{~m}, 1 \mathrm{H}$, one of $\left.\mathrm{CH}_{2}-5\right), 1.35\left(\mathrm{~d}, J=7.6 \mathrm{~Hz}, 3 \mathrm{H}, \mathrm{CH}_{3}-11\right), 1.88\left(\mathrm{dd}, J=13.6\right.$ and $4.9 \mathrm{~Hz}, 1 \mathrm{H}$, one of $\left.\mathrm{CH}_{2}-5\right), 2.40-2.44$ $(\mathrm{m}, 1 \mathrm{H}, \mathrm{H}-7), 2.45-2.49(\mathrm{~m}, 1 \mathrm{H}, \mathrm{H}-6), 3.40\left(\mathrm{~d}, J=2.4 \mathrm{~Hz}, 2 \mathrm{H}, \mathrm{CH}_{2}-10\right), 4.84(\mathrm{dd}, J=5.0$ and $4.8 \mathrm{~Hz}, 1 \mathrm{H}$, $\mathrm{H}-1), 5.82(\mathrm{~d}, J=10.0 \mathrm{~Hz}, 1 \mathrm{H}, \mathrm{H}-3), 5.94(\mathrm{dd}, J=10.0$ and $3.9 \mathrm{~Hz}, 1 \mathrm{H}, \mathrm{H}-2) ;{ }^{13} \mathrm{C}-\mathrm{NMR}: 15.3$ (C-11), 24.8 (C-9), 33.5 (C-5), 37.3 (C-4), 38.6 (C-7), 42.9 (C-6), 69.2 (C-10), 73.12 (C-1), 123.01 (C-2), 139.9 (C-3), 179.8 (C-8); IR (KBr, cm $\left.{ }^{-1}\right)$ : 3468, 2964, 1785, 1456, 1170, 1024; ESIHRMS: calcd. for $\mathrm{C}_{11} \mathrm{H}_{16} \mathrm{O}_{3}, \mathrm{~m} / z$ z 197.1178 $(\mathrm{M}+\mathrm{H})^{+}$, found 197.1181. (Supplementary Materials, Figures S35-S40).

2,3-Epoxy-4,4,7-trimethyl-9-oxabicyclo[4.3.0]nonan-8-one (9a) was characterized by the following physical and spectral properties: colourless oil; ${ }^{1} \mathrm{H}-\mathrm{NMR}: 0.85$ (dd, $J=13.7$ and $13.7 \mathrm{~Hz}, 1 \mathrm{H}$, one of $\mathrm{CH}_{2}-5 \mathrm{~B}$ ), 1.05 (s, 3H, CH $3-9 \mathrm{~B}), 1.08$ (s, 3H, $\left.\mathrm{CH}_{3}-9 \mathrm{~A}\right), 1.15$ (d, J = 7.1 Hz, 3H, $\left.\mathrm{CH}_{3}-11 \mathrm{~B}\right), 1.17\left(\mathrm{~s}, 6 \mathrm{H}, \mathrm{CH}_{3}-10 \mathrm{~A}\right.$, $\left.\mathrm{CH}_{3}-10 \mathrm{~B}\right), 1.18-1.19\left(\mathrm{~m}, 1 \mathrm{H}\right.$, one of $\left.\mathrm{CH}_{2}-5 \mathrm{~B}\right), 1.20-1.21\left(\mathrm{~m}, 2 \mathrm{H}, \mathrm{CH}_{2}-5 \mathrm{~A}\right), 1.32(\mathrm{~d}, J=7.5 \mathrm{~Hz}, 3 \mathrm{H}$, $\left.\mathrm{CH}_{3}-11 \mathrm{~A}\right), 12.15-2.20(\mathrm{~m}, 1 \mathrm{H}, \mathrm{H}-6 \mathrm{~A}), 2.41-2.47(\mathrm{~m}, 2 \mathrm{H}, \mathrm{H}-6 \mathrm{~B}$ and $\mathrm{H}-7 \mathrm{~A}), 2.84$ (quintet, $J=7.1 \mathrm{~Hz}, 1 \mathrm{H}$, H-7B), $3.02(\mathrm{~d}, J=3.2 \mathrm{~Hz}, 1 \mathrm{H}, \mathrm{H}-3 \mathrm{~A}), 3.04(\mathrm{~d}, J=3.3 \mathrm{~Hz}, 1 \mathrm{H}, \mathrm{H}-3 \mathrm{~B}), 3.46(\mathrm{~d}, J=3.2 \mathrm{~Hz}, 1 \mathrm{H}, \mathrm{H}-2 \mathrm{~A})$, $3.52(\mathrm{dd}, J=3.3$ and $1.3 \mathrm{~Hz}, 1 \mathrm{H}, \mathrm{H}-2 \mathrm{~B}), 4.67(\mathrm{~d}, J=4.2 \mathrm{~Hz}, 1 \mathrm{H}, \mathrm{H}-1 \mathrm{~B}), 4.82(\mathrm{~d}, J=6.1 \mathrm{~Hz}, 1 \mathrm{H}, \mathrm{H}-1 \mathrm{~A})$; ${ }^{13}$ C-NMR: 9.2 (C-11B), 14.4 (C-11A), 23.4 (C-9B), 25.4 (C-9A), 28.3 (C-4B), 28.8 (C-10A), 28. 8 (C-4A), 30.0 (C-10B), 33.2 (C-5B), 34.4 (C-6B), 35.7 (C-5A), 27.6 (C-6A), 39.9 (C-7B), 41.4 (C-7A), 52.3 (C-2B), 52.4 (C-2A), 61.1 (C-3A), 61.2 (C-3B), 73.5 (C-A), 74.1 (C-1B), 178.2 (C-8A), 178.2 (C-8B); IR (KBr, cm $\left.{ }^{-1}\right)$ : 2952, 1785, 1476, 1457, 1160, 1024; ESIHRMS: calcd. for $\mathrm{C}_{11} \mathrm{H}_{16} \mathrm{O}_{3}, m / z 197.1178[\mathrm{M}+\mathrm{H}]^{+}$, found 197.1179. (Supplementary Materials, Figures S41-S46)

1-Hydroxy-4,4,6,7-tetramethyl-9-oxabicyclo[4.3.0]non-2-en-8-one (6b) was characterized by the following physical and spectral properties: colourless oil; ${ }^{1} \mathrm{H}-\mathrm{NMR}: 1.13\left(\mathrm{~d}, J=7.0 \mathrm{~Hz}, 3 \mathrm{H}, \mathrm{CH}_{3}-11 \mathrm{~A}\right), 1.20(\mathrm{~s}, 3 \mathrm{H}$, $\left.\mathrm{CH}_{3}-9 \mathrm{~A}\right), 1.21$ (s, 3H, $\left.\mathrm{CH}_{3}-9 \mathrm{~B}\right), 1.23$ (d, J = 7.5 Hz, 3H, $\left.\mathrm{CH}_{3}-11 \mathrm{~B}\right), 1.28\left(\mathrm{~s}, 6 \mathrm{H}, \mathrm{CH}_{3}-10 \mathrm{~B}, \mathrm{CH}_{3}-12 \mathrm{~B}\right), 1.30$ $\left(\mathrm{s}, 3 \mathrm{H}, \mathrm{CH}_{3}-10 \mathrm{~A}\right), 1.34\left(\mathrm{~s}, 3 \mathrm{H}, \mathrm{CH}_{3}-12 \mathrm{~A}\right), 1.65\left(\mathrm{~d}, J=14.1 \mathrm{~Hz}, 1 \mathrm{H}\right.$, one of $\left.\mathrm{CH}_{2}-5 \mathrm{~B}\right), 1.86-1.89(\mathrm{~m}, 1 \mathrm{H}$, $\left.\mathrm{CH}_{2}-5 \mathrm{~A}\right), 2.02-2.05\left(\mathrm{~m}, 1 \mathrm{H}\right.$, one of $\left.\mathrm{CH}_{2}-5 \mathrm{~A}\right), 2.19-2.22\left(\mathrm{~m}, 1 \mathrm{H}\right.$, one of $\left.\mathrm{CH}_{2}-5 \mathrm{~B}\right), 3.01-3.02(\mathrm{~m}, 1 \mathrm{H}, \mathrm{H}-7 \mathrm{~B})$, 3.12-3.13 (m, 1H, H-7A), $5.86(\mathrm{~d}, J=9.9 \mathrm{~Hz}, 1 \mathrm{H}, \mathrm{H}-3 \mathrm{~A}), 5.89(\mathrm{~d}, J=10.1 \mathrm{~Hz}, 1 \mathrm{H}, \mathrm{H}-3 \mathrm{~A}), 6.60-6.62(\mathrm{~m}$, 2H, H-2A, H-2B); ${ }^{13} \mathrm{C}-\mathrm{NMR}: 10.9$ (C-11B), 12.5 (C-11A), 24.5 (C-12A), 24.3 (C-10A), 28.8 (C-10B), 29.2 (C-12B), 32.2 (C-9B), 32.4 (C-9A), 40.8 (C-5A), 42.1 (C-5B), 44.7 (C-7B), 45.0 (C-7A), 44.7 (C-4A), 45.0 (C-4B), 44.4 (C-6A), 44.7 (C-6B), 124.4 (C-3A), 124.7 (C-3B), 157.3 (C-2B), 157.3 (C-2A), 178.1 (C-1B), 178.2 (C-1A), 178.2 (C-8A), 179.6 (C-8B); IR (KBr, cm $\left.{ }^{-1}\right)$ : 3480, 2962, 1751, 1675, 1461, 1382, 1242, 1154, 1065; ESIHRMS: calcd. for $\mathrm{C}_{11} \mathrm{H}_{18} \mathrm{O}_{3}, m / z 211.1334[\mathrm{M}+\mathrm{H}]^{+}$, found 211.1330. (Supplementary Materials, Figures S83-S88). 
7-Hydroxy-4,4,6,7-tetramethyl-9-oxabicyclo[4.3.0]non-2-en-8-one (7b) was characterized by the following physical and spectral properties: colourless oil; ${ }^{1} \mathrm{H}-\mathrm{NMR}: 1.05\left(\mathrm{~s}, 3 \mathrm{H}, \mathrm{CH}_{3}-9\right), 1.15-1.16(\mathrm{~m}, 1 \mathrm{H}$, one of $\left.\mathrm{CH}_{2}-5\right), 1.17\left(\mathrm{~s}, 3 \mathrm{H}, \mathrm{CH}_{3}-10\right), 1.19\left(\mathrm{~s}, 3 \mathrm{H}, \mathrm{CH}_{3}-11\right), 1.32\left(\mathrm{~s}, 3 \mathrm{H}, \mathrm{CH}_{3}-12\right), 1.33-1.36(\mathrm{~m}, 1 \mathrm{H}$, one of $\left.\mathrm{CH}_{2}-5\right), 4.67(\mathrm{~d}, J=5.3 \mathrm{~Hz}, 1 \mathrm{H}, \mathrm{H}-1), 5.80(\mathrm{dd}, J=10.0$ and $5.3 \mathrm{~Hz}, 1 \mathrm{H}, \mathrm{H}-2), 5.90(\mathrm{~d}, J=10.0 \mathrm{~Hz}, 1 \mathrm{H}$, H-3); ${ }^{13}$ C-NMR: 14.7 (C-11), 16.9 (C-12), 17.0 (C-4), 28.8 (C-9), 32.3 (C-6), 33.2 (C-10), 40.0 (C-5), 43.0 (C-7), 77.3 (C-1), 117.4 (C-2), 144.4 (C-3), 177.7 (C-8B); IR (KBr, cm ${ }^{-1}$ ): 2464, 2927, 1757, 1464, 1382, 1141, 974; ESIHRMS: calcd. for $\mathrm{C}_{11} \mathrm{H}_{18} \mathrm{O}_{3}, m / z 211.1334[\mathrm{M}+\mathrm{H}]^{+}$, found 211.1331, calcd. for $\mathrm{C}_{11} \mathrm{H}_{18} \mathrm{O}_{3} \mathrm{Na}$, $m / z 233.1154[\mathrm{M}+\mathrm{H}]^{+}$, found 233.1166. (Supplementary Materials, Figures S89-S94).

10-Hydroxy-4,4,6,7-tetramethyl-9-oxabicyclo[4.3.0]non-2-en-8-one (8b) was characterized by the following physical and spectral properties: colourless oil; ${ }^{1} \mathrm{H}-\mathrm{NMR}: 1.06\left(\mathrm{~s}, 3 \mathrm{H}, \mathrm{CH}_{3}-9\right), 1.09$ (s, 3H, $\left.\mathrm{CH}_{3}-12\right), 1.12$ $\left(\mathrm{d}, J=7.3 \mathrm{~Hz}, 3 \mathrm{H}, \mathrm{CH}_{3}-11\right), 1.57\left(\mathrm{~d}, J=15.0 \mathrm{~Hz}, 1 \mathrm{H}\right.$, one of $\left.\mathrm{CH}_{2}-5\right), 1.80(\mathrm{~d}, J=15.0 \mathrm{~Hz}, 1 \mathrm{H}$, one of $\left.\mathrm{CH}_{2}-5\right), 2.80(\mathrm{q}, J=7.3 \mathrm{~Hz}, 1 \mathrm{H}, \mathrm{H}-7), 3.29\left(\mathrm{~d}, J=10.5 \mathrm{~Hz}, 1 \mathrm{H}\right.$, one of $\left.\mathrm{CH}_{2}-10\right), 3.38(\mathrm{~d}, J=10.5 \mathrm{~Hz}, 1 \mathrm{H}$, one of $\left.\mathrm{CH}_{2}-10\right), 4.53(\mathrm{~s}, 1 \mathrm{H}, \mathrm{H}-1), 5.64(\mathrm{dd}, J=10.4$ and $5.3 \mathrm{~Hz}, 1 \mathrm{H}, \mathrm{H}-2), 5.79$ (d, J = 10.4 Hz, 1H, H-3); ${ }^{13}$ C-NMR: 7.2 (C-11), 23.2 (C-9), 24.1 (C-12), 36.03 (C-5), 37.2 (C-4), 40.4 (C-6), 40.8 (C-7), 72.0 (C-10), 80.9 (C-1), 125.2 (C-3), 135.8 (C-2), 178.7 (C-8B); IR (KBr, cm $\left.{ }^{-1}\right)$ : 3409, 2933, 1757, 1451, 1207, 1175, 1041; ESIHRMS: calcd. for $\mathrm{C}_{11} \mathrm{H}_{18} \mathrm{O}_{3}, m / z 211.1334[\mathrm{M}+\mathrm{H}]^{+}$, found 211.1331, calcd. for $\mathrm{C}_{11} \mathrm{H}_{18} \mathrm{O}_{3} \mathrm{Na}, \mathrm{m} / z$ 233.1154 $[\mathrm{M}+\mathrm{H}]^{+}$, found 233.1019. (Supplementary Materials, Figures S95-S100).

2,3-Epoxy-4,4,6,7-tetramethyl-9-oxabicyclo[4.3.0]nonan-8-one (9b) was characterized by the following physical and spectral properties: colourless oil; ${ }^{1} \mathrm{H}-\mathrm{NMR}$ : 0.98 (s, 6H, $\left.\mathrm{CH}_{3}-9 \mathrm{~A}, \mathrm{CH}_{3}-9 \mathrm{~B}\right), 1.08$ (d, $\left.J=7.2 \mathrm{~Hz}, 3 \mathrm{H}, \mathrm{CH}_{3}-11 \mathrm{~A}\right), 1.10\left(\mathrm{~d}, J=7.2 \mathrm{~Hz}, 3 \mathrm{H}, \mathrm{CH}_{3}-11 \mathrm{~B}\right), 1.12\left(\mathrm{~s}, 3 \mathrm{H}, \mathrm{CH}_{3}-12 \mathrm{~A}\right), 1.16(\mathrm{~s}, 3 \mathrm{H}$, $\left.\mathrm{CH}_{3}-12 \mathrm{~B}\right), 1.21$ (s, 6H, $\left.\mathrm{CH}_{3}-10 \mathrm{~A}, \mathrm{CH}_{3}-10 \mathrm{~B}\right), 1.26-1.30\left(\mathrm{dd}, J=15.1\right.$ and $\left.9.9 \mathrm{~Hz}, 2 \mathrm{H}, \mathrm{CH}_{2}-5 \mathrm{~A}\right), 1.37(\mathrm{~d}$, $\left.J=15.1 \mathrm{~Hz}, 2 \mathrm{H}, \mathrm{CH}_{2}-5 \mathrm{~B}\right), 2.37(\mathrm{q}, J=7.2 \mathrm{~Hz}, 1 \mathrm{H}, \mathrm{H}-7 \mathrm{~A}), 2.80(\mathrm{q}, J=7.2 \mathrm{~Hz}, 1 \mathrm{H}, \mathrm{H}-7 \mathrm{~B}), 2.98(\mathrm{~d}, J=3.3 \mathrm{~Hz}$, 1H, H-3B), 3.02 (d, $J=3.5 \mathrm{~Hz}, 1 \mathrm{H}, \mathrm{H}-3 \mathrm{~A}), 3.33(\mathrm{~d}, J=3.3 \mathrm{~Hz}, 1 \mathrm{H}, \mathrm{H}-2 \mathrm{~B}), 3.60(\mathrm{dd}, J=3.5$ and $3.3 \mathrm{~Hz}$, 1H, H-2A), 4.29 (s, 1H, H-1B), 4.42 (m, 1H, H-1A); ${ }^{13} \mathrm{C}-\mathrm{NMR}: 6.9$ (C-11A), 7.4 (C-11B), 23.5 (C-9A), 23.6 (C-9B), 26.5 (C-5B), 26.6 (C-5A), 29.5 (C-6B), 30.1 (C-12B), 31.1 (C-12A), 33.1 (C-6A), 38.0 (C-10A), 38.2 (C-10B), 39.6 (C-4B), 39.9 (C-7B), 41.1 (C-4A), 49.9 (C-7A), 53.4 (C-2A), 53.8 (C-2B), 60.7 (C-3B), 60.8 (C-3A), 78.3 (C-1A), 79.3 (C-1B), 178.2 (C-8A), 178.2 (C-8B); IR (KBr, cm $\left.{ }^{-1}\right)$ : 2963, 1707, 1673, 1459, 1383, 1241, 1096; ESIHRMS: calcd. for $\mathrm{C}_{11} \mathrm{H}_{18} \mathrm{O}_{3}, m / z 211.1334[\mathrm{M}+\mathrm{H}]^{+}$, found 211.1331, calcd. for $\mathrm{C}_{11} \mathrm{H}_{18} \mathrm{O}_{3} \mathrm{Na}, \mathrm{m} / z 233.1154[\mathrm{M}+\mathrm{H}]^{+}$, found 233.1019. (Supplementary Materials, Figures S101-S106).

\section{Conclusions}

Two new bicyclic unsaturated lactones $\mathbf{5 a}$ and $\mathbf{5 b}$, obtained as a pairs of diastereoisomers $\mathbf{A}$ and B after a four-step synthesis, were subjected to a screening biotransformation using fourteen fungal strains. Some of them were able to introduce a hydroxy group or an oxirane ring into the molecules of lactones $\mathbf{5 a}$ and $\mathbf{5 b}$. During these biotransformations eight new compounds were obtained: six hydroxylactones and two epoxylactones. In both substrates the hydroxy group was introduced onto a tertiary carbon (allylic position) or onto a primary carbon. Unexpectedly, hydroxylation of other non-allylic tertiary carbons (C-6 for $\mathbf{5 a}$ and C-7 for $\mathbf{5 b}$ ) was also observed. The products with hydroxy groups in an allylic position and epoxylactones were created as pairs of diastereoisomers $\mathbf{A}$ and $\mathbf{B}$. Four other products were formed as single isomers (only B). Among these compounds, lactones $7 \mathbf{a}$ were obtained with a predominance of the (-)-isomer, while in the case of lactones $\mathbf{7 b}, \mathbf{8} \mathbf{a}$ and $\mathbf{8 b}$, the (+)-isomer was formed preferentially.

Supplementary Materials: Supplementary materials can be accessed at: http:/ /www.mdpi.com/1420-3049/22/ $1 / 147 / \mathrm{s} 1$.

Acknowledgments: Publication supported by the Wroclaw Centre of Biotechnology under the Leading National Research Centre (KNOW) programme for years 2014-2018.

Author Contributions: Katarzyna Wińska conceived and designed the experiments and analyzed the data; Katarzyna Wińska, Wanda Mączka and Adrianna Kondas performed the experiments; Katarzyna Wińska and Małgorzata Grabarczyk wrote the paper; Gabriela Maciejewska performed the high resolution mass spectrometry 
analysis; Małgorzata Grabarczyk analyzed NMR spectrum of products; and Mirosław Anioł contributed to the discussion of results. Katarzyna Wińska, Małgorzata Grabarczyk, Radosław Bonikowski performed the GC analysis. All authors read and approved the final manuscript.

Conflicts of Interest: The authors declare no conflict of interest.

\section{References}

1. Nazari, Z.E.; Iranshahi, M. Biologically active sesquiterpene coumarins from Ferula species. Phytother. Res. 2011, 25, 315-323. [CrossRef] [PubMed]

2. Zhang, H.; Conte, M.M.; Capon, R.J. Franklinolides A-C from an Australian marine sponge complex: Phosphodiesters strongly enhance polyketide cytotoxicity. Angew. Chem. Int. Ed. 2010, 49, 9904-9906. [CrossRef] [PubMed]

3. Chen, L.H.; Fang, J.; Li, H.; Demark-Wahnefried, W.; Lin, X. Enterolactone induces apoptosis in human prostate carcinoma LNCaP cells via a mitochondrial-mediated, caspase-dependent pathway. Mol. Cancer Ther. 2007, 6, 2581-2590. [CrossRef] [PubMed]

4. Kwon, H.C.; Kauffman, C.A.; Jensen, P.R.; Fenical, W. Marinisporolides, polyene-polyol macrolides from a marine actinomycete of the new genus Marinispora. J. Org. Chem. 2009, 74, 675-684. [CrossRef] [PubMed]

5. Ohguchi, K.; Ito, M.; Yokoyama, K.; Iinuma, M.; Itoh, T.; Nozawa, Y.; Akao, Y. Effects of sesquiterpene lactones on melanogenesis in mouse B16 melanoma cells. Biol. Pharm. Bull. 2009, 32, 308-310. [CrossRef] [PubMed]

6. He, Z.; Zhang, A.; Ding, L.; Lei, X.; Sun, J.; Zhang, L. Chemical composition of the green alga Codium divaricatum Holmes. Fitoterapia 2010, 81, 1125-1128. [CrossRef] [PubMed]

7. Sassa, T.; Ooi, T.; Kinoshita, H. Isolation and structures of acremolactones B and C, novel plant-growth inhibitory gamma-lactones from Acremonium roseum I4267. Biosci. Biotechnol. Biochem. 2004, 68, 2633-2636. [CrossRef] [PubMed]

8. Chen, V.X.; Boyer, F.D.; Rameau, C.; Retailleau, P.; Vors, J.P.; Beau, J.M. Stereochemistry, total synthesis, and biological evaluation of the new plant hormone solanacol. Chemistry 2010, 16, 13941-13945. [CrossRef] [PubMed]

9. Wanzola, M.; Furuta, T.; Kohno, Y.; Fukumitsu, S.; Yasukochi, S.; Watari, K.; Tanaka, C.; Higuchi, R.; Miyamoto, T. Four new cembrane diterpenes isolated from an Okinawan soft coral Lobophytum crassum with inhibitory effects on nitric oxide production. Chem. Pharm. Bull. 2010, 58, 1203-1209. [CrossRef] [PubMed]

10. Grabarczyk, M.; Wińska, K.; Mączka, W.; Potaniec, B.; Anioł, M. Loliolide-The most ubiquitous lactone. Folia Biol. Oecol. 2015, 11, 1-8. [CrossRef]

11. Ma, X.C.; Zheng, J.; Guo, D.A. Microbial transformation of dehydrocostuslactone and costunolide by Mucor polymorphosporus and Aspergillus candidus. Enzym. Microb. Technol. 2007, 40, 1013-1019. [CrossRef]

12. Grudniewska, A.; Wawrzeńczyk, C. Lactones 41. Synthesis and microbial hydroxylation of unsaturated terpenoid lactones with p-menthane ring systems. Molecules 2013, 18, 2778-2787. [CrossRef] [PubMed]

13. Bhatti, H.N.; Khan, S.S.; Khan, A.; Rani, M.; Ahmad, V.U.; Choudhary, M.I. Biotransformation of monoterpenoids and their antimicrobial activities. Phytomedicine 2014, 21, 1597-1626. [CrossRef] [PubMed]

14. Nunes, F.M.; dos Santos, G.F.; Saraiva, N.N.; Trap, M.A.; de Mattos, M.C.; da Conceicao, M.; Oliveira, F.; Rodrigues-Filho, E. New fungi for whole-cell biotransformation of carvone enantiomers. Novel p-menthane2,8,9-triols production. Appl. Catal. A 2013, 468, 88-94. [CrossRef]

15. Gładkowski, W.; Grabarczyk, M.; Wińska, K.; Ratuś, B.; Białońska, A.; Ciunik, Z.; Wawrzeńczyk, C. Lactones 26 [1]: Stereoselective microbial epoxidation of unsaturated bicyclic $\gamma$-lactones with the alkylsubstituted cyclohexane system. J. Mol. Catal. B Enzym. 2007, 49, 79-87. [CrossRef]

16. Martinez, A.; Perojil, A.; Rivas, F.; Parra, A.; Garcia-Granados, A.; Fernandez-Vivas, A. Biotransformation of oleanolic and maslinic methyl esters by Rhizomucor miehei CECT 2749. Phytochemistry 2015, 117, 500-508. [CrossRef] [PubMed]

17. Hu, S.; Sun, D.; Tian, X.; Fang, Q. Selective microbial hydroxylation and biological rearrangement of taxoids. Tetrahedron Lett. 1997, 38, 2721-2724. [CrossRef]

18. Roiban, G.D.; Agudo, R.; Reetz, M.T. Stereo- and regioselectivity in the P450-catalyzed oxidative tandem difunctionalization of 1-methylcyclohexene. Tetrahedron 2013, 69, 5306-5311. [CrossRef] 
19. Gandomkar, S.; Habibi, Z. Biotransformation of $6 \alpha$-santonin and 1,2-dihydro- $\alpha$-santonin by Acremonium chrysogenum PTCC 5271 and Rhizomucor pusillus PTCC 5134. J. Mol. Catal. B Enzym. 2014, 110, 59-63. [CrossRef]

20. Ning, L.; Zhan, J.; Qu, G.; Zhong, L.; Guo, H.; Bi, K.; Gu, D. Biotransformation of triptolide by Cunninghamella blakesleana. Tetrahedron 2003, 59, 4209-4213. [CrossRef]

21. Chen, Z.; Li, J.; Lin, H.; Shao, L.; Qin, J.; Fan, X.; Dong, X.; Chen, D. Biotransformation of 14-deoxy-14-methylenetriptolide into a novel hydroxylation product by Neurospora crassa. J. Biosci. Bioeng. 2013, 116, 199-202. [CrossRef] [PubMed]

22. Gładkowski, W.; Grabarczyk, M.; Wińska, K.; Białońska, A.; Ciunik, Z.; Wawrzeńczyk, C. Lactones 27 [1]: Transformations of $\gamma$-lactones fused to a dimethylcyclohexane ring in Absidia cylindrospora cultures. J. Mol. Catal. B Enzym. 2006, 39, 31-39. [CrossRef]

23. Wińska, K.; Grabarczyk, M.; Mączka, W.; Żarowska, B.; Maciejewska, G.; Anioł, M. Antimicrobial activity of new bicyclic lactones with three or four methyl groups obtained both synthetically and biosynthetically. J. Saudi Chem. Soc. 2016. [CrossRef]

24. Wińska, K.; Grabarczyk, M.; Mączka, W.; Żarowska, B.; Maciejewska, G.; Anioł, M. Influence of structure of lactones with the methylcyclohexene and dimethylcyclohexene ring on their biotransformation and antimicrobial activity. Z. Naturforsch. C 2017, in press.

25. Johnson, W.S.; Werthemann, L.; Bartlett, W.R.; Brocksom, T.J.; Li, T.T.; Faulkner, D.J.; Petersen, M.R. Simple stereoselective version of the Claisen rearrangement leading to trans-trisubstituted olefinic bonds. Synthesis of squalene. J. Am. Chem. Soc. 1970, 92, 741-743. [CrossRef]

26. Wawrzeńczyk, C.; Lochyński, S. Synthesis of juvenoids with the 5,5-dimethylcyclohexane system. Monatsch. Chem. 1985, 116, 99-110. [CrossRef]

27. Loupy, A.; Seyden-Penne, J. The influence of lithium complexing agents on the regioselectivity of reductions of substituted 2-cyclohexenones by LiAlH4 and LiBH4. Tetrahedron 1980, 36, 1937-1942. [CrossRef]

28. Grabarczyk, M.; Szumny, A.; Gładkowski, W.; Białońska, A.; Ciunik, Z.; Wawrzeńczyk, C. Lactones 18. Synthesis of bicyclic lactones with methyl-, di- and trimethyl substituted cyclohexane system. Pol. J. Chem. 2005, 79, 1763-1771. [CrossRef]

Sample Availability: Samples of the compounds $\mathbf{1} \mathbf{a}, \mathbf{b}-\mathbf{9} \mathbf{a}, \mathbf{b}$ are available from the authors.

(c) 2017 by the authors; licensee MDPI, Basel, Switzerland. This article is an open access article distributed under the terms and conditions of the Creative Commons Attribution (CC-BY) license (http://creativecommons.org/licenses/by/4.0/). 